Published in "Israel Journal of Mathematics 159(1): 373-411, 2007"

which should be cited to refer to this work.

\title{
THE NORMAL CYCLE OF A COMPACT DEFINABLE SET
}

\author{
BY \\ AndREAS BERNIG* \\ Département de Mathématiques \\ Chemin du Musée 23, 1700 Fribourg, Switzerland \\ e-mail: andreas.bernig@unifr.ch
}

\begin{abstract}
An elementary construction of the normal cycle of a compact definable set in Euclidean space (and more generally of a compactly supported constructible function) is given. Here "definable" means definable in some o-minimal structure. The construction is based on the notion of support function and uses only basic o-minimal geometry.
\end{abstract}

\section{Introduction}

The normal cycle of a compact subanalytic set in a finite-dimensional Euclidean vector space was constructed by J. Fu in 1994 [15]. The primary motivation was to generalize certain curvature notions to non-smooth sets. Since then, the normal cycle turned out to be useful in a number of applications. J. Fu introduced and studied Lipschitz-Killing curvatures of subanalytic spaces. A variational formula for Lipschitz-Killing curvatures, which implies several versions of the Schläfli differential formula, was proved in [5]. Based on this work, several tensor-valued curvature measures were introduced in [7].

The normal cycle is an important tool in the study of valuations on Euclidean spaces and even on arbitrary manifolds. By a recent theorem of Alesker [1], each $G L(V)$-smooth valuation on a Euclidean vector space $V$ can be represented as integration of a differential form against the normal cycle. This was used in the proof of a Hard Lefschetz Theorem for translation invariant valuations in

\footnotetext{
* Supported by the Schweizerischer Nationalfonds grant SNF 200020-105010/1.
} 
[9]. Smooth valuations on manifolds were studied by Alesker [1], [2], [3] and Alesker-Fu [4] where the normal cycle of differentiable polyhedra is used.

As one application of the (co-) normal cycle in topology we mention the construction of Chern classes of complex varieties [14] and of Stiefel-Whitney classes [17]. Recently, the normal cycle was used to define curvature measures for certain fractal sets [23].

Fu's construction of the normal cycle is based on Geometric Measure Theory. The subanalytic set is approximated by some easier sets and, with the help of the Federer-Fleming compactness theorem, a convergent subsequence of currents was extracted. The hard part of the proof is a uniqueness theorem, which shows that the limit current is independent of the choices.

A sheaf-theoretical construction of the normal cycle of a subanalytic set (under the name characteristic cycle) was provided at about the same time by Kashiwara and Shapira [22].

Another construction, using Stratified Morse Theory, was given by Bröcker and Kuppe [11]. It has the advantage of describing the normal cycle more explicitly in terms of Morse indices. The drawback of this construction is that it is difficult to see that the resulting current is a cycle and, moreover, that it is really the normal cycle. Both problems can be solved with Fu's uniqueness theorem.

The goal of this paper is to provide a self-contained construction of the normal cycle which does not rely on other theories like Geometric Measure Theory, Sheaf Theory or Stratified Morse Theory. By doing so, we hope to make it easier for subanalytic geometers to understand and use this important object. Our construction only uses easy results about subanalytic sets, like cell-decompositions and finiteness properties. In fact, the construction also works for all sets which belong to some o-minimal structure in the sense of [28] (they will be called definable for short) and even for so-called constructible functions.

The outline of the construction is as follows. Let $V$ be a finite-dimensional Euclidean vector space with unit sphere $S(V)$ and sphere bundle $S V:=V \times S(V)$. The key notion of the construction is that of support function. In Convex Geometry, one associates a homogeneous real-valued function to each compact convex set in the Euclidean vector space $V$, its support function. By work of Bröcker [10], one can also provide constructible functions on $V$ with a support function. In the first section, we will recall this construction. The support function is not a real-valued function, but a function with values in the group ring $\mathbb{Z}[\mathbb{R}]$. Our first result, contained in Section 3, is that the support 
function of a constructible function $\phi$ is Lipschitz (with respect to the flat norm on $\mathbb{Z}[\mathbb{R}])$ if and only if the support of $\phi$ is bounded.

We denote by $\mathcal{C}_{c}(V)$ the set of compactly supported constructible functions. The set of definable, $\mathbb{Z}[\mathbb{R}]$-valued Lipschitz continuous functions on the sphere $S(V)$ is denoted by $\mathcal{D}_{\text {Lip }}(S(V), \mathbb{Z}[\mathbb{R}])$. With this notation, the first result is that there is a bijection

$$
\mathcal{C}_{c}(V) \stackrel{\cong}{\longrightarrow} \mathcal{D}_{L i p}(S(V), \mathbb{Z}[\mathbb{R}])
$$

As was remarked in [8], a compactly supported Legendrian cycle in the sphere bundle $S V$ also admits a support function. Since its construction is based on Slicing Theory, it is only defined almost everywhere. With $\mathcal{L C}_{c}(S V)$ denoting the space of compactly supported Legendrian cycles on $S V$, the support function induces an injective map

$$
\mathcal{L C}_{c}(S V) \rightarrow L^{1}(S(V), \mathbb{Z}[\mathbb{R}])
$$

In the special case we are interested in, the current is definable and the slicing is easier. In Section 4, we will recall the definition of definable currents. We state without proof Hardt's slicing theorem, which will only be used in the study of the properties of the normal cycle, but not in its construction. Our second result, contained in Section 5, is that one can extend the support function of a compactly supported definable Legendrian cycle by (Lipschitz-) continuity. Stated otherwise, the image of the restriction of the map $\mathcal{L C}_{c}(S V) \rightarrow L^{1}(S(V), \mathbb{Z}[\mathbb{R}])$ to definable currents is contained in $\mathcal{D}_{L i p}(S(V), \mathbb{Z}[\mathbb{R}])$. If a compactly supported definable Legendrian cycle $T$ has the same support function as a compactly supported constructible function $\phi$, we say that $T$ is the normal cycle of $\phi$.

From these results, it follows that each compactly supported definable Legendrian cycle is the normal cycle of some compactly supported constructible function. Conversely, given a compactly supported constructible function, we will construct in Section 6 its normal cycle. Since the homogeneous extension of the support function is piecewise $C^{1}$, we can first construct the "graph of the differential" of the support function, which is a conical Lagrangian current. In general, it is not closed and we have to "fill the holes" in order to obtain a conical Lagrangian cycle.

It follows that the support function provides an isomorphism between the space of compactly supported definable Legendrian cycles on $S V$ and $\mathcal{D}_{L i p}(S(V), \mathbb{Z}[\mathbb{R}])$, the latter space being isomorphic to $\mathcal{C}_{c}(V)$. 
The reader should keep in mind the following commutative diagram

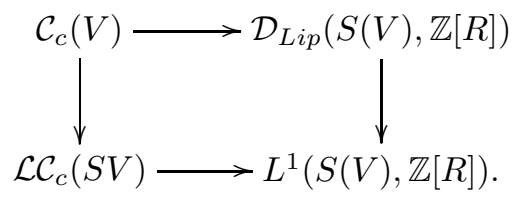

The horizontal arrows are the support function constructions, the vertical arrow on the left is the normal cycle construction and the vertical arrow on the right is the inclusion of the set of Lipschitz continuous constructible functions in $L^{1}(S(V), \mathbb{Z}[\mathbb{R}])$.

ACKnowledgement: This paper is a short version of my Habilitationsschrift [6]. I wish to thank the University of Fribourg, in particular R. Kellerhals, for their hospitality. I also thank L. Bröcker and G. Comte for stimulating discussions.

\section{Support functions of constructible functions}

2.1. O-minimal systems. Let us recall the definition of an o-minimal system.

Definition 2.1: An o-minimal system is a collection $\mathcal{M}=\left(\mathcal{M}_{n}\right), n \in \mathbb{Z}$, where each $\mathcal{M}_{n}$ is a Boolean subalgebra of the power set of $\mathbb{R}^{n}$ such that the following axioms are satisfied:

(1) algebraic subsets of $\mathbb{R}^{n}$ belong to $\mathcal{M}_{n}$;

(2) if $X \in \mathcal{M}_{n}, Y \in \mathcal{M}_{m}$ then $X \times Y \in \mathcal{M}_{n+m}$;

(3) if $\pi: \mathbb{R}^{n+1} \rightarrow \mathbb{R}^{n}$ denotes the projection on the first $n$ coordinates and $X \in \mathcal{M}_{n+1}$, then $\pi(X) \in \mathcal{M}_{n} ;$

(4) $\mathcal{M}_{1}$ consists precisely of finite unions of points and intervals.

Examples of o-minimal sets comprise the set of semialgebraic subsets, globally subanalytic sets or sets definable in $\mathcal{R}_{a n \text {,exp }}$, i.e. by means of the exponential function. See [28] for details.

In the following, we will fix an o-minimal system $\mathcal{M}$. By a definable set we mean a set $X \subset \mathbb{R}^{n}$ which belongs to $\mathcal{M}_{n}$. Recall also that, given a definable set $D \subset \mathbb{R}^{n}$, a function $f: D \rightarrow \mathbb{R}^{m}$ is called definable if its graph is a definable subset of $\mathbb{R}^{n} \times \mathbb{R}^{m}$. From Axiom (3) we infer that the image of a definable set under a definable function is again definable. 
Definition 2.2: Let $k \in \mathbb{N}$. A definable $C^{k}$-cell decomposition of $\mathbb{R}$ is a partition of $\mathbb{R}$ in finitely many cells, which are points (of dimension 0 ) or open intervals (dimension 1).

A definable $C^{k}$-cell decomposition of $\mathbb{R}^{n}, n>1$ is given by a $C^{k}$-cell decomposition of $\mathbb{R}^{n-1}$ and, for each cell $D$ of $\mathbb{R}^{n-1}$, finitely many definable $C^{k}$-functions

$$
\xi_{D, 1}<\cdots<\xi_{D, l(d)}: D \rightarrow \mathbb{R}
$$

The cells are the graphs (of dimension $\operatorname{dim} D$ )

$$
\left\{\left(x, \xi_{D, i}(x)\right) \in \mathbb{R}^{n-1} \times \mathbb{R}: x \in D\right\}, \quad i=1, \ldots, l(D)
$$

and the (open) bands of dimension $\operatorname{dim} D+1$

$$
\left\{(x, y) \in \mathbb{R}^{n} \times \mathbb{R}: x \in D, \xi_{D, i}(x)<y<\xi_{D, i+1}(x)\right\}, \quad i=0, \ldots, l(D)
$$

where $\xi_{D, 0}=-\infty, \xi_{D, l(D)+1}=\infty$.

Theorem 2.3 ( $C^{k}$-cell decomposition of definable sets): Given finitely many definable subsets $X_{1}, \ldots, X_{m}$ of $\mathbb{R}^{n}$ and $k \in \mathbb{Z}$, there exists a definable $C^{k}$-cell decomposition of $\mathbb{R}^{n}$ compatible with $X_{i}, i=1, \ldots, m$ (i.e. each such set is a union of cells).

We refer to [12] for the proof.

Definition 2.4: A function $\phi: \mathbb{R}^{n} \rightarrow \mathbb{Z}$ is called constructible if the range of $\phi$ is finite and $\phi^{-1}(a)$ is definable for each $a \in \mathbb{Z}$. A function $\phi: X \rightarrow \mathbb{Z}$ on a definable set $X \subset \mathbb{R}^{n}$ is called constructible, if its extension by 0 is constructible.

A definable subset $X \subset \mathbb{R}^{n}$ can be identified with its characteristic function, which is constructible. The restriction of $\phi$ to a definable subset $X$ will be denoted by $\phi \cap X$.

\subsection{EUler integration.}

Definition And Proposition 2.5: Let $X \subset \mathbb{R}^{n}$ be definable. Choose a $C^{0}$-cell decomposition of $\mathbb{R}^{n}$ such that $X$ is a union of cells. Then the number

$$
\chi(X):=\sum_{D \subset X}(-1)^{\operatorname{dim} D}
$$

is independent of the choice of the cell decomposition and called Euler characteristic of $X$ (in fact, $\chi(X)$ is the Euler characteristic with respect to BorelMoore homology). The Euler characteristic of a constructible function $\phi: V \rightarrow \mathbb{Z}$ 
is defined by

$$
\chi(\phi):=\sum_{a \in \mathbb{Z}} a \chi\left(\phi^{-1}(a)\right) .
$$

We will also write $\int_{\mathbb{R}^{n}} \phi(x) d \chi(x)$ instead of $\chi(\phi)$ and $\int_{X} \phi(x) d \chi(x)$ instead of $\chi(\phi \cap X)$.

THEOREM 2.6 (Fubini for Euler characteristic): Let $X \subset \mathbb{R}^{n}$ be definable and let $\phi: X \rightarrow \mathbb{Z}$ be a constructible function. Given a definable function $f: X \rightarrow \mathbb{R}^{m}$, the push-forward $f_{*} \phi$, defined by

$$
f_{*} \phi(y):=\chi\left(f^{-1}(y) \cap \phi\right), \quad y \in \mathbb{R}^{m}
$$

is a constructible function on $\mathbb{R}^{m}$. Moreover,

$$
\int_{X} \phi(x) d \chi(x)=\int_{\mathbb{R}^{m}} f_{*} \phi(y) d \chi(y)
$$

The proof is easy using a cell-decomposition of the graph of $f$.

Corollary 2.7: Let $\phi: \mathbb{R}^{n} \times \mathbb{R}^{n} \rightarrow \mathbb{Z}$ be constructible. Then

$$
\int_{\mathbb{R}^{n}} \int_{\mathbb{R}^{n}} \phi(x, y) d \chi(x) d \chi(y)=\int_{\mathbb{R}^{n}} \int_{\mathbb{R}^{n}} \phi(x, y) d \chi(y) d \chi(x) .
$$

Definition 2.8: The convolution of two constructible functions $\phi$ and $\psi$ on $\mathbb{R}^{n}$ is the constructible function $\phi * \psi$ defined by

$$
\phi * \psi(x):=\int_{\mathbb{R}^{n}} \phi(y) \psi(x-y) d \chi(y)
$$

The set of constructible functions on $\mathbb{R}^{n}$, endowed with addition + and multiplication $*$, is a commutative ring with unit $1_{\{0\}}$. Its prime ideals, units etc. were studied by Bröcker [10].

Definition 2.9: Let $\phi$ be a constructible function on $\mathbb{R}^{n}$ and $\psi$ a constructible function on $\mathbb{R}^{m}$. Then the exterior product $\phi \otimes \psi$ is the constructible function on $\mathbb{R}^{n} \times \mathbb{R}^{m}$ defined by

$$
\phi \otimes \psi\left(x_{1}, x_{2}\right)=\phi\left(x_{1}\right) \psi\left(x_{2}\right) \quad x_{1} \in \mathbb{R}^{n}, x_{2} \in \mathbb{R}^{m}
$$


2.3. Support FunCtions. The group ring $\mathbb{Z}[\mathbb{R}]$ is the set of finite linear combinations $\sum_{i=1}^{k} a_{i} \delta_{r_{i}}$, where $a_{i} \in \mathbb{Z}$ and $r_{i} \in \mathbb{R}$ are pairwise different. The sum of two such elements is defined in the obvious way, and the multiplication is given by the convolution product:

$$
\left(\sum_{i=1}^{k} a_{i} \delta_{r_{i}}\right) \cdot\left(\sum_{j=1}^{l} b_{j} \delta_{s_{j}}\right)=\sum_{i=1}^{k} \sum_{j=1}^{l} a_{i} b_{j} \delta_{r_{i}+s_{j}} .
$$

Elements of $\mathbb{Z}[\mathbb{R}]$ can be considered as integer multiplicity rectifiable 0 -currents on $\mathbb{R}$ (compare with [13]). If $T=\sum_{i=1}^{k} a_{i} \delta_{r_{i}}$ and $f \in C_{c}^{\infty}(\mathbb{R})$, then $T(f):=$ $\sum_{i=1}^{k} a_{i} f\left(r_{i}\right)$. The mass of $T$ is $\mathbf{M}(T):=\sum_{i=1}^{k}\left|a_{i}\right|$ and its flat norm is

$$
\mathbf{F}(T):=\sup \left\{T(f): f \in C_{c}^{\infty}(\mathbb{R}):\|f\|_{\infty} \leq 1,\left\|f^{\prime}\right\|_{\infty} \leq 1\right\} .
$$

The augmentation of $T$ is the integer $T(1)=\sum_{i=1}^{k} a_{i}$. We can identify $\mathbb{R}$ with a subset of $\mathbb{Z}[\mathbb{R}]$ by sending $x$ to $\delta_{x}$.

Proposition 2.10: Let $\phi$ be a constructible function on $\mathbb{R}$.

(1)

$$
\sum_{x \in \mathbb{R}} \lim _{s \rightarrow 0^{+}}(\phi(x)-\phi(x+s)) \delta_{x}
$$

is an element of $\mathbb{Z}[\mathbb{R}]$, denoted by $\phi^{\prime}$ and called jump of $\phi$.

(2) If $\phi$ has compact support, then

$$
\phi^{\prime}(1)=\chi(\phi) .
$$

(3) If $\phi$ is continuous from the left, then

$$
\int_{\mathbb{R}} \phi(s) d \chi(s)=-\lim _{s \rightarrow \infty} \phi(s) .
$$

Proof: Since $\phi$ is constructible, there exists a finite partition of $\mathbb{R}$ into points and open intervals, such that $\phi$ is constant on each cell. If $x$ belongs to an open interval, then the coefficient before $\delta_{x}$ vanishes, from which (1) follows. Statement (2) is easily verified. If $\phi$ is continuous from the left, then $\phi$ is constant on finitely many half-open intervals of the from $(a, b]$ (where $a=-\infty$ is possible) and on one open interval $(a, \infty)$. Since $\chi((a, b])=0, \chi((a, \infty))=-1$, (3) follows.

In the following, $V$ denotes an $n$-dimensional Euclidean vector space. After choice of an orthogonal basis, $V$ can be identified with $\mathbb{R}^{n}$. The notions definable subset and constructible function are independent of the choice of this basis. 
Definition 2.11: Let $\phi$ be a constructible function on $V$. For each $y \in V$ let $\pi_{y}: V \rightarrow \mathbb{R}, x \mapsto\langle x, y\rangle$ and define

$$
h_{\phi}(y):=\left(\left(\pi_{y}\right)_{*} \phi\right)^{\prime} \in \mathbb{Z}[\mathbb{R}]
$$

The function

$$
h_{\phi}: V \rightarrow \mathbb{Z}[\mathbb{R}]
$$

is called support function of $\phi$.

Proposition 2.12:

(1) $h_{\phi}(0)=\chi(\phi) \delta_{0}$.

(2) $h_{\phi}$ is homogeneous in the following sense: if $\lambda \geq 0$, then

$$
h_{\phi}(\lambda y)=\left(m_{\lambda}\right)_{*}\left(h_{\phi}(y)\right),
$$

where $\left(m_{\lambda}\right)_{*}\left(\sum_{i} a_{i} \delta_{r_{i}}\right):=\sum_{i} a_{i} \delta_{\lambda r_{i}}$. Therefore, we can identify $h_{\phi}$ with its restriction to $S(V)$.

(3) If $\phi$ has compact support, then the augmentation of $h_{\phi}(y)$ equals $\chi(\phi)$ for all $y \in V$.

(4) Given $A \in G L(V)$, define $A_{*} \phi$ by $A_{*} \phi(x):=\phi\left(A^{-1} x\right)$. Then

$$
h_{A_{*} \phi}(y)=h_{\phi}\left(A^{*} y\right) \text {. }
$$

(5) For constructible functions $\phi$ and $\psi$ on $V$,

$$
\begin{aligned}
h_{\phi+\psi} & =h_{\phi}+h_{\psi} \\
h_{\phi * \psi} & =h_{\phi} \cdot h_{\psi} .
\end{aligned}
$$

(6) Let $W$ be a Euclidean vector space. For a constructible function $\phi$ on $V$ and a constructible function $\psi$ on $W$,

$$
h_{\phi \otimes \psi}\left(y_{1}, y_{2}\right)=h_{\phi}\left(y_{1}\right) \cdot h_{\psi}\left(y_{2}\right) \quad \forall y_{1} \in V, y_{2} \in W .
$$

(7) Let $\phi$ be a constructible function on $V$. Let $W$ be a linear subspace and $\pi: V \rightarrow W$ the orthogonal projection. Then $\pi_{*} \phi$ is a constructible function on $W$ and

$$
h_{\pi_{*} \phi}=\left.h_{\phi}\right|_{W} .
$$

Proof: Using Fubini's Theorem, the proofs are easy. 
Example: Let $K \subset V$ be a compact (definable) convex set and $\phi:=1_{K}$ its characteristic function. If $y \in V$, then the push-forward $\left(\pi_{y}\right)_{*} \phi$ is the characteristic function of the compact interval $\left[\min _{x \in K}\langle x, y\rangle, \max _{x \in K}\langle x, y\rangle\right]$. The jump of this function is given by $\delta_{\max _{x \in K}\langle x, y\rangle}$. The function mapping $y$ to $\max _{x \in K}\langle x, y\rangle$ is the classical support function of $K$ (compare with [26]). Therefore, the support function of $1_{K}$ is the same (using the embedding $\mathbb{R} \rightarrow \mathbb{Z}[\mathbb{R}]$ ) as the classical support function of $K$.

Definition 2.13: A function $h: \mathbb{R}^{n} \rightarrow \mathbb{Z}[\mathbb{R}]$ is called definable if the function

$$
\mathbb{R}^{n} \times \mathbb{R} \rightarrow \mathbb{Z}, \quad(y, r) \mapsto h(y)(r)
$$

is constructible. Here $h(y)(r)$ denotes the coefficient of $\delta_{r}$ in $h(y) \in \mathbb{Z}[\mathbb{R}]$.

Proposition 2.14: If $\phi: V \rightarrow \mathbb{Z}$ is constructible, then $h_{\phi}: V \rightarrow \mathbb{Z}[\mathbb{R}]$ is definable.

Proof: Again, this is an easy consequence of Theorems 2.3 and 2.6.

Theorem 2.15: A function $h: V \rightarrow \mathbb{Z}[\mathbb{R}]$ is the support function of a constructible function $\phi$ on $V$ if and only if $h$ is definable and homogeneous.

The "only if"-part is contained in Proposition 2.12, (2) and Proposition 2.14. In [10] one finds the proof of the "if"-part. Below, we will prove a similar statement.

\section{Lipschitz continuity of support functions}

Theorem 3.1: A function $h: V \rightarrow \mathbb{Z}[\mathbb{R}]$ is the support function of a compactly supported constructible function $\phi$ on $V$ if and only if $h$ is definable, homogeneous and Lipschitz with respect to $\mathbf{F}$. In this case, $\phi$ is unique.

Proof (Compact support implies Lipschitz): Suppose $h=h_{\phi}$ is the support function of a constructible function $\phi$ on $V$. By Proposition 2.12, (2) and Proposition 2.14, $h$ is homogeneous and definable.

Suppose that the support of $\phi$ is contained in a compact set, say $\operatorname{spt} \phi \subset$ $B(0, R), R>0$. Since $h$ is definable, there is an $M>0$ with

$$
\mathbf{M}(h(y)) \leq M \quad \forall y \in V .
$$

We claim that $h$ is $6 M R$-Lipschitz with respect to F. By Proposition 2.12 (7), it is enough to show this in the case $\operatorname{dim} V=2$. We can assume furthermore that $\phi$ is not constantly 0 . 
It suffices to prove that every $y \in V$ has a neighborhood $U$ such that

$$
\mathbf{F}\left(h\left(y^{\prime}\right)-h(y)\right) \leq 6 M R\left\|y^{\prime}-y\right\| \quad \text { for all } y^{\prime} \in U .
$$

We fix an orthogonal basis of $V$ and identify $V$ with $\mathbb{R}^{2}$.

First, suppose that $y=0$. Then $h(0)=\chi(\phi) \delta_{0}$ by $2.12(1) ; h\left(y^{\prime}\right)=\sum_{i=1}^{k} a_{i} \delta_{r_{i}}$ with $\sum_{i=1}^{k} a_{i}=\chi(\phi)(2.12(3)), \sum_{i=1}^{k}\left|a_{i}\right| \leq M$ and $\left|r_{i}\right| \leq R\left\|y^{\prime}\right\|$.

Then

$$
\mathbf{F}\left(\sum_{i=1}^{k} a_{i} \delta_{r_{i}}-\chi(\phi) \delta_{0}\right) \leq \sum_{i=1}^{k}\left|a_{i}\right| \mathbf{F}\left(\delta_{r_{i}}-\delta_{0}\right) \leq \sum_{i=1}^{k}\left|a_{i}\right| R\left\|y^{\prime}\right\| \leq M R\left\|y^{\prime}\right\| .
$$

Next we suppose that $y \neq 0$. Using homogeneity, we can assume, without loss of generality, that $y=(1,0)$.

By Theorem 2.3, there exists a $C^{2}$-cell decomposition of $\mathbb{R}^{2}$ such that $\phi$ is constant on each cell. We can refine the decomposition and assume that each of the functions $\xi_{D, i}, i=1, \ldots, l(D)$, where $D$ runs over the cells of $\mathbb{R}$, is convex or concave.

Lemma 3.2: If $\xi: I \rightarrow \mathbb{R}$ is a convex or concave $C^{2}$-function on a bounded open interval $I \subset \mathbb{R}$ such that $\operatorname{graph}(\xi) \subset B(0, R)$, then for $s \in I$

$$
\left|\xi^{\prime}(s)\right| \leq \frac{2 R}{d(s, \partial I)} .
$$

Proof: Easy exercise.

Since $\phi$ has compact support, it is non-zero only on finitely many, bounded cells. Fix a number $0<\rho_{\max }<1 / 2$ such that $12 R \rho_{\max }$ is smaller than the lengths of the cells in $\mathbb{R}$ above which $\phi$ is non-zero.

Lemma 3.3: Let $y^{\prime}=\left(y_{1}^{\prime}, y_{2}^{\prime}\right) \in \mathbb{R}^{2}$ with $\rho:=\left\|y-y^{\prime}\right\|<\rho_{\max }$. Let $t \in D$, where $D$ is an open cell of $\mathbb{R}$ and suppose $d(t, \partial D)>\epsilon:=6 \rho R$. Then each intersection of the line $L_{t}=L_{t}\left(y^{\prime}\right)$ defined by

$$
L_{t}=\left\{\left(x_{1}, x_{2}\right) \in \mathbb{R}^{2}: y_{1}^{\prime} x_{1}+y_{2}^{\prime} x_{2}=t\right\}
$$

with a cell contained in $B(0, R)$ is empty or transversal.

Proof: Let $D=(a, b)$, where $a=-\infty$ or $b=\infty$ is possible.

Let $\left(x_{1}, x_{2}\right) \in B(0, R)$ be the intersection of $L_{t}$ with a cell.

Then

$$
\rho R \geq\left|y_{2}^{\prime} x_{2}\right|=\left|t-x_{1}+x_{1}-y_{1}^{\prime} x_{1}\right| \geq\left|t-x_{1}\right|-\left|x_{1}\left(1-y_{1}^{\prime}\right)\right| \geq\left|t-x_{1}\right|-\rho R,
$$


which implies $\left|t-x_{1}\right| \leq 2 \rho R=\epsilon / 3$.

It follows that $x_{1} \in D$ and $d\left(x_{1}, \partial D\right)>2 / 3 \epsilon$.

The cell in which $\left(x_{1}, x_{2}\right)$ lies is either a band or a graph of a function $\xi=\xi_{D, i}$. In the first case, the intersection is trivially transversal.

In the second case,

$$
\left|\xi^{\prime}\left(x_{1}\right)\right| \leq \frac{2 R}{d\left(x_{1}, \partial D\right)}<\frac{3 R}{\epsilon}=\frac{1}{2 \rho} .
$$

If the intersection is non-transversal, then $y_{2}^{\prime} \neq 0$ and

$$
\frac{1}{2 \rho} \leq \frac{\left|y_{1}^{\prime}\right|}{\left|y_{2}^{\prime}\right|}=\left|\xi^{\prime}\left(x_{1}\right)\right|<\frac{1}{2 \rho}
$$

a contradiction.

Let $r_{1}<\cdots<r_{k}$ be the endpoints of the cells of $\mathbb{R}$. Denote by $\pi$ the projection of $\mathbb{R}^{2}$ to the first coordinate. Since the Euler characteristic of $\pi^{-1}(s) \cap \phi, s \in \mathbb{R}$ is constant on each cell, $h_{\phi}(y)$ is concentrated on $\left\{r_{1}, \ldots, r_{k}\right\}$, say $h_{\phi}(y)=$ $\sum_{i=1}^{k} a_{i} \delta_{r_{i}}$.

Denote $\pi^{\prime}: \mathbb{R}^{2} \rightarrow \mathbb{R}, x \mapsto\left\langle x, y^{\prime}\right\rangle$. Let $t \in \mathbb{R}$ be at distance at least $\epsilon$ from $\left\{r_{1}, \ldots, r_{k}\right\}$. By Lemma 3.3, $\left(\pi^{\prime}\right)^{-1}(t)=L_{t}\left(y^{\prime}\right)$ intersects the cell decomposition transversally, which implies that

$$
\chi\left(\left(\pi^{\prime}\right)^{-1}(t)\right)=\chi\left(\pi^{-1}(t)\right) .
$$

It follows that, if $h_{\phi}\left(y^{\prime}\right)=\sum_{j=1}^{l} b_{j} \delta_{s_{j}}$, then the $s_{j}$ are contained in the open $\epsilon$-neighborhood of $\left\{r_{1}, \ldots, r_{k}\right\}$. Note that the $\epsilon$-neighborhoods of the different $r_{i}$ are disjoint by choice of $\epsilon$.

From Theorem 2.6 and Proposition 2.10, applied to the function

$$
\left[r_{i}-\epsilon, r_{i}+\epsilon\right) \rightarrow \mathbb{Z}, s \mapsto \chi\left(\pi^{-1}(s) \cap \phi\right)
$$

we infer that

$$
\chi\left(\pi^{-1}\left[r_{i}-\epsilon, r+\epsilon\right) \cap \phi\right)=a_{i} .
$$

In the same way,

$$
\chi\left(\left(\pi^{\prime}\right)^{-1}\left[r_{i}-\epsilon, r+\epsilon\right) \cap \phi\right)=\sum_{j:\left|s_{j}-r_{i}\right| \leq \epsilon} b_{j} .
$$

The intersection of the strip $\pi^{-1}\left[r_{i}-\epsilon, r+\epsilon\right)$ with the cell decomposition is transversal by Lemma 3.3. The same is true for all $y^{\prime \prime}$ on the line between $y$ 
and $y^{\prime}$. By simple counting (or by applying Thom's isotopy lemma, [18], [25]), we obtain that the Euler characteristics are equal, which means that

$$
\sum_{j:\left|s_{j}-r_{i}\right| \leq \epsilon} b_{j}=a_{i} .
$$

Using $\mathbf{F}\left(\delta_{t}-\delta_{s}\right) \leq|s-t|$ for reals $s, t$, we get that

$$
\begin{aligned}
\mathbf{F}\left(h_{\phi}\left(y^{\prime}\right)-h_{\phi}(y)\right) & =\mathbf{F}\left(\sum_{i=1}^{k} \sum_{j:\left|s_{j}-r_{i}\right| \leq \epsilon}\left(b_{j} \delta_{s_{j}}-b_{j} \delta_{r_{i}}\right)\right) \\
& \leq \sum_{i=1}^{k} \sum_{j:\left|s_{j}-r_{i}\right| \leq \epsilon} \underbrace{\mathbf{F}\left(b_{j} \delta_{s_{j}}-b_{j} \delta_{r_{i}}\right)}_{\leq \varepsilon\left|b_{j}\right|} \\
& \leq \epsilon \sum_{j=1}^{l}\left|b_{j}\right| \\
& =\epsilon \mathbf{M}\left(h_{\phi}\left(y^{\prime}\right)\right) \\
& \leq 6 M R\left\|y^{\prime}-y\right\| .
\end{aligned}
$$

We deduce that $h$ is $6 M R$-Lipschitz.

Remark: For later use we note the following. Let $\phi: V \rightarrow \mathbb{Z}$ be a constructible function with support in $B(0, R)$. Let $D \subset V$ be a $C^{2}$-cell such that $h=h_{\phi}$ is given on $D$ by

$$
h(y)=\sum_{i=1}^{k} a_{i} \delta_{f_{i}(y)}
$$

with definable $C^{2}$-functions $f_{i}: D \rightarrow \mathbb{R}, f_{1}<\cdots<f_{k}$ and non-zero natural numbers $a_{i}$. Then the above argument shows that the norm of the gradient of each $f_{i}$ is bounded by $6 R$.

Uniqueness: Let $h=h_{\phi}$ be the support function of a compactly supported constructible function $\phi$. Then $\chi(\phi)=h(y)(1)$ for all $y \in V$, in particular $\chi(\phi)$ can be computed from $h$.

We compute that, for all $t \in \mathbb{R}$,

$$
h(y)(-\infty, t)=\int_{V} \phi(x) 1_{\left\{x^{\prime} \in V:\left\langle x^{\prime}, y\right\rangle<t\right\}}(x) d \chi(x) .
$$

Since $h$ is definable, the function

$$
\psi_{x}(y):=h(y)(-\infty,\langle x, y\rangle)
$$


is constructible.

It easily follows from Fubini's Theorem that

$$
\phi(x)=\chi(\phi)+(-1)^{n-1} \int_{V} \psi_{x}(y) d \chi(y) .
$$

This holds true for all $x \in V$ and thus $\phi$ is unique.

Lipschitz implies compact support: Suppose that $h: V \rightarrow \mathbb{Z}[\mathbb{R}]$ is definable, homogeneous and Lipschitz with Lipschitz constant $L>0$. We will show that $h$ is the support function of a constructible function with support in $B(0, L)$.

STEP 1: We claim that $\operatorname{spt} h(y) \subset[-L\|y\|, L\|y\|]$ for all $y \in V$. To prove the claim, fix $y \in V$ and suppose that $h(y)=\sum_{i=1}^{k} a_{i} \delta_{r_{i}}$ with $a_{i} \in \mathbb{Z}, a_{i} \neq 0$ and $r_{1}<r_{2}<\cdots<r_{k}$. Suppose $r_{k}>0$.

Fix a real number $\lambda>1$ such that $\lambda r_{k-1}<r_{k}$ and $(\lambda-1) r_{k}<1$. Let $f$ be a piecewise affine function which equals 0 on $\left(-\infty, r_{k}\right]$, grows linearly on $\left[r_{k}, \lambda r_{k}\right]$, equals 1 at $\lambda r_{k}$ and which is symmetric with respect to the center $\lambda r_{k}$. By homogeneity, $h(\lambda y)=\sum_{i=1}^{k} a_{i} \delta_{\lambda r_{i}}$ and therefore $h(\lambda y)(f)=a_{k}, h(y)(f)=0$. Approximating $f$ by compactly supported smooth functions and using that $h$ is $L$-Lipschitz with respect to $\mathcal{F}$, we obtain

$$
\left|a_{k}\right|=|h(\lambda y)(f)-h(y)(f)| \leq L\|\lambda y-y\| \max \{\underbrace{\|f\|_{\infty}}_{=1}, \underbrace{\left\|f^{\prime}\right\|_{\infty}}_{=\frac{1}{(\lambda-1) r_{k}}}\}=L\|y\| / r_{k} .
$$

We deduce that $r_{k} \leq L\|y\|$ and similarly $r_{1} \geq-L\|y\|$.

STEP 2: With $h$ being Lipschitz, the value $a:=h(y)(1) \in \mathbb{Z}$ is independent of $y \in V$.

Since $h$ is definable, the function

$$
\psi_{x}(y):=h(y)(-\infty,\langle x, y\rangle)
$$

is constructible.

We have seen in the uniqueness proof that if there exists $\phi$ with $h_{\phi}=h$, then $\phi$ has to be given by

$$
\phi(x):=a+(-1)^{n-1} \int_{V} \psi_{x}(y) d \chi(y) .
$$

We claim that indeed $h_{\phi}=h$.

Given $v_{0} \in S(V)$ and $t_{0} \in \mathbb{R}$, we set

$$
W_{0}:=\left\{x \in V:\left\langle x, v_{0}\right\rangle=t_{0}\right\}
$$


Fubini's theorem shows that

$$
\int_{W_{0}} \int_{V} \psi_{x}(y) d \chi(y) d \chi(x)=\int_{V} \int_{W_{0}} \psi_{x}(y) d \chi(x) d \chi(y) .
$$

We evaluate the inner integral and consider several cases for $y$.

(1) $y=0$. By homogeneity, $h(0)=a \delta_{0}$ and thus $\psi_{x}(0)=0$ for all $x$. It follows

$$
\int_{W_{0}} \psi_{x}(0) d \chi(x)=0
$$

(2) $y \| v_{0}, y \neq 0$. For $\lambda>0$ we obtain

$$
\begin{aligned}
\psi_{x}\left(\lambda v_{0}\right) & =h\left(\lambda v_{0}\right)\left(-\infty,\left\langle x, \lambda v_{0}\right\rangle\right)=h\left(v_{0}\right)\left(-\infty, t_{0}\right) \\
\psi_{x}\left(-\lambda v_{0}\right) & =h\left(-v_{0}\right)\left(-\infty,-t_{0}\right) .
\end{aligned}
$$

and thus

$$
\begin{aligned}
\int_{W_{0}} \psi_{x}\left(\lambda v_{0}\right) d \chi(x) & =(-1)^{n-1} h\left(v_{0}\right)\left(-\infty, t_{0}\right) \\
\int_{W_{0}} \psi_{x}\left(-\lambda v_{0}\right) d \chi(x) & =(-1)^{n-1} h\left(-v_{0}\right)\left(-\infty,-t_{0}\right) .
\end{aligned}
$$

(3) $y \not \mid v_{0}$. With $y^{\perp}:=y-\left\langle y, v_{0}\right\rangle v_{0}$ we get $\left\langle y^{\perp}, v_{0}\right\rangle=0$ and $\left\langle y^{\perp}, y\right\rangle>0$.

For $x_{0} \in W_{0}$, the line $l:=\left\{x_{0}+s y^{\perp}: s \in \mathbb{R}\right\}$ is contained in $W_{0}$ and we compute, using Proposition 2.10, (3),

$$
\begin{aligned}
\int_{l} \psi_{x}(y) d \chi(x) & =\int_{\mathbb{R}} \psi_{x_{0}+s y^{\perp}}(y) d \chi(s) \\
& =\int_{\mathbb{R}} h(y)\left(-\infty,\left\langle x_{0}+s y^{\perp}, y\right\rangle\right) d \chi(s) \\
& =\int_{\mathbb{R}} h(y)\left(-\infty, s^{\prime}\right) d \chi\left(s^{\prime}\right) \\
& =-a .
\end{aligned}
$$

This is true for all lines in $W_{0}$ parallel to $y^{\perp}$ and implies, by Fubini's theorem,

$$
\int_{W_{0}} \psi_{x}(y) d \chi(x)=(-1)^{n-1} a .
$$

From these considerations, we deduce that

$$
\begin{aligned}
\int_{V} \int_{W_{0}} \psi_{x}(y) d \chi(x) d \chi(y)= & (-1)^{n}\left(h\left(v_{0}\right)\left(-\infty, t_{0}\right)\right. \\
& \left.+h\left(-v_{0}\right)\left(-\infty,-t_{0}\right)\right)+\left(1+(-1)^{n}\right)(-1)^{n-1} a .
\end{aligned}
$$


It follows that

$$
\int_{W_{0}} \phi(x) d \chi(x)=a-h\left(v_{0}\right)\left(-\infty, t_{0}\right)-h\left(-v_{0}\right)\left(-\infty,-t_{0}\right),
$$

from which we easily deduce that $h_{\phi}=h$ on $S(V)$, and then, by homogeneity of both sides, on $V$.

\section{STEP 3:}

Lemma 3.4: Let $D \subset V$ be a $C^{1}$-cell and let $h: D \rightarrow \mathbb{Z}[\mathbb{R}]$ be given by $h(y)=$ $\sum_{i=1}^{k} a_{i} \delta_{f_{i}(y)}$ with real-valued $C^{1}$-functions $f_{1}<\cdots<f_{k}$ on $D$ and non-zero integers $a_{i}$. If $h$ is $L$-Lipschitz with respect to $\mathbf{F}$, then $\left\|\operatorname{grad} f_{i}(y)\right\| \leq L$ for all $y \in D$ and $i=1, \ldots, k$.

Proof: Fix $y \in D$ and $i \in\{1, \ldots, k\}$ and set $c:=f_{i}(y)$. Let $1>\eta>0$ be smaller than the minimum of $f_{i+1}(y)-f_{i}(y)$ and $f_{i}(y)-f_{i-1}(y)$ (if $i=1$ or $i=k$ or even $k=1$ then the corresponding difference will be set to be $\infty$ ).

By continuity of the $f_{i}$, there exists a neighborhood $U \subset D$ of $y$ such that $f_{i+1}\left(y^{\prime}\right)>c+\eta, f_{i-1}\left(y^{\prime}\right)<c-\eta$ and $c-\eta \leq f_{i}\left(y^{\prime}\right) \leq c+\eta$ for $y^{\prime} \in U$.

Define a piecewise affine function $g$ on $\mathbb{R}$ by

$$
g(z)= \begin{cases}1-\frac{|z-c|}{\eta} & z \in[c-\eta, c+\eta] \\ 0 & \text { otherwise }\end{cases}
$$

Since $h$ is $L$-Lipschitz and since $\|g\|_{\infty}=1<1 / \eta,\left\|g^{\prime}\right\|_{\infty}=1 / \eta$, we get for all $y^{\prime} \in U$

$$
\begin{aligned}
\frac{\left|a_{i}\right|}{\eta}\left|f_{i}\left(y^{\prime}\right)-f_{i}(y)\right| & =\left|a_{i} g\left(f_{i}\left(y^{\prime}\right)\right)-a_{i} g\left(f_{i}(y)\right)\right|=\left|h(y)(g)-h\left(y^{\prime}\right)(g)\right| \\
& \leq \frac{L}{\eta}\left\|y^{\prime}-y\right\| .
\end{aligned}
$$

It follows that $\left\|\operatorname{grad} f_{i}\right\| \leq L / a_{i} \leq L$.

STEP 4: Let $x \in V$ with $t:=\|x\|>L$. Write $x=t v_{0}$ with $v_{0} \in S(V)$ and fix $y_{0} \in V$.

We claim that the function $g: \mathbb{R} \rightarrow \mathbb{Z}$ defined by

$$
g(s):=h\left(y_{0}+s v_{0}\right)(-\infty, \underbrace{\left\langle x, y_{0}+s v_{0}\right\rangle}_{\left\langle x, y_{0}\right\rangle+s t})
$$

is continuous from the left. 
The function $s \mapsto h\left(y_{0}+s v_{0}\right)$ is definable and $L$-Lipschitz by hypothesis. Fix $s_{0} \in \mathbb{R}$. For all $s$ in some interval $\left(s_{0}-\epsilon, s_{0}\right)$, we get

$$
h\left(y_{0}+s v_{0}\right)=\sum_{i=1}^{k} a_{i} \delta_{f_{i}(s)},
$$

with definable, real-valued $C^{1}$-functions $f_{1}<\cdots<f_{k}$. Lemma 3.4 implies that they are $L$-Lipschitz. Each $f_{i}$ is bounded (compare with Step 1 ) and can be extended by continuity to $s_{0}$. Then we also have $h\left(y_{0}+s_{0} v_{0}\right)=\sum_{i=1}^{k} a_{i} \delta_{f_{i}\left(s_{0}\right)}$.

It follows that

$$
g(s)=\sum_{i: f_{i}(s)<\left\langle x, y_{0}\right\rangle+s t} a_{i}
$$

for all $s \in\left(s_{0}-\epsilon, s_{0}\right]$.

If $f_{i}\left(s_{0}\right) \neq c:=\left\langle x, y_{0}\right\rangle+s_{0} t$, then either $f_{i}(s)<\left\langle x, y_{0}\right\rangle+s t$ or $\left.f_{i}(s)\right\rangle$ $\left\langle x, y_{0}\right\rangle+s t$ for all $s$ in some (maybe smaller) interval $\left(s_{0}-\epsilon, s_{0}\right]$.

If $f_{i}\left(s_{0}\right)=c$, then, since $f_{i}$ is $L$-Lipschitz, for all $s<s_{0}$ near $s_{0}$

$$
f_{i}(s) \geq f_{i}\left(s_{0}\right)+L\left(s-s_{0}\right)=\left\langle x, y_{0}\right\rangle+s t+(t-L)\left(s_{0}-s\right) \geq\left\langle x, y_{0}\right\rangle+s t .
$$

We deduce that $g(s)=g\left(s_{0}\right)$ for all $s<s_{0}$ near $s_{0}$, which proves the claim.

SteP 5: The function $g$ from Step 4 is continuous from the left and satisfies $\lim _{s \rightarrow \infty} g(s)=a$, since spt $h\left(y_{0}+s v_{0}\right) \subset\left(-\infty,\left\langle x, y_{0}+s v_{0}\right\rangle\right)$ for large $s$ (compare with Step 1).

With $l:=\left\{y_{0}+s v_{0}: s \in \mathbb{R}\right\}$, and using Proposition 2.10 (3), we get

$$
\int_{l} \psi_{x}(y) d \chi(y)=\int_{\mathbb{R}} g(s) d \chi(s)=-a .
$$

The same holds for every line parallel to $v_{0}$. Fubini's theorem implies that

$$
\phi(x)=a+(-1)^{n-1} \int_{V} \psi_{x}(y) d \chi(y)=0 .
$$

Therefore the support of $\phi$ is contained in $B(0, L)$.

\section{Definable Legendrian cycles}

4.1. Definable Currents. Let $\mathcal{D}^{k}\left(\mathbb{R}^{n}\right)$ denote the space of $k$-forms with compact support on $\mathbb{R}^{n}$. The topology of $\mathcal{D}^{k}\left(\mathbb{R}^{n}\right)$ is the usual one, which is characterized by the fact that a sequence $\omega^{1}, \omega^{2}, \ldots \in \mathcal{D}^{k}\left(\mathbb{R}^{n}\right)$ converges to 
$\omega \in \mathcal{D}^{k}\left(\mathbb{R}^{n}\right)$ if and only if there is a compact set $K \subset \mathbb{R}^{n}$ such that the supports of $\omega^{1}, \ldots$ are contained in $K$ and such that all partial derivatives (of arbitrary degree) of the coefficients of $\omega^{j}$ converge uniformly to the corresponding derivatives of the coefficients of $\omega$.

Definition 4.1: A continuous functional $T: \mathcal{D}^{k}\left(\mathbb{R}^{n}\right) \rightarrow \mathbb{R}$ is called a (FedererFleming-) $k$-current on $\mathbb{R}^{n}$. The space of $k$-currents is denoted by $\mathcal{D}_{k}\left(\mathbb{R}^{n}\right)=$ $\left(\mathcal{D}^{k}\left(\mathbb{R}^{n}\right)\right)^{*}$.

The boundary $\partial T$ of $T \in \mathcal{D}_{k}\left(\mathbb{R}^{n}\right)$ is the current $\partial T \in \mathcal{D}_{k-1}\left(\mathbb{R}^{n}\right)$ defined by

$$
\partial T(\omega)=T(d \omega) \quad \forall \omega \in \mathcal{D}^{k-1}\left(\mathbb{R}^{n}\right) .
$$

$T$ is called a cycle if $\partial T=0$.

The restriction of $T \in \mathcal{D}_{k}\left(\mathbb{R}^{n}\right)$ to a form $\xi \in \mathcal{D}^{l}\left(\mathbb{R}^{n}\right), l \leq k$ is the current $T\left\llcorner\xi \in \mathcal{D}^{k-l}\left(\mathbb{R}^{n}\right)\right.$ with

$$
T\left\llcorner\xi(\omega)=T(\xi \wedge \omega) \quad \forall \omega \in \mathcal{D}^{k-l}\left(\mathbb{R}^{n}\right) .\right.
$$

The support of $T \in \mathcal{D}_{k}\left(\mathbb{R}^{n}\right)$ is the closed set

$\operatorname{spt} T:=\left\{\bigcap_{K \subset \mathbb{R}^{n}} K\right.$ closed $: T(\omega)=0$ for all $\omega \in \mathcal{D}^{k}\left(\mathbb{R}^{n}\right)$ with spt $\left.\omega \subset \mathbb{R}^{n} \backslash K\right\}$.

Example: An oriented, $k$-dimensional $C^{1}$-manifold $M$ with boundary $\partial M$ defines a $k$-current $[[M]] \in \mathcal{D}_{k}\left(\mathbb{R}^{n}\right)$ by

$$
[[M]](\omega)=\int_{M} \omega, \quad \omega \in \mathcal{D}^{k}\left(\mathbb{R}^{n}\right) .
$$

Stokes's theorem implies that

$$
\partial[[M]]=[[\partial M]]
$$

In the same way, oriented $k$-dimensional cells of a $C^{1}$-cell decomposition of $\mathbb{R}^{n}$ define $k$-currents whose boundaries are given by integration over $k-1$ dimensional cells.

Definition 4.2: A current $T \in \mathcal{D}_{k}\left(\mathbb{R}^{n}\right)$ is called definable if there exists a definable $C^{1}$-cell decomposition and for each $k$-cell $D$ an orientation of $D$ and a number $n_{D}$ such that

$$
T=\sum_{D} n_{D}[[D]]
$$


The mass of $T$ is defined by

$$
\mathbf{M}(T):=\sum_{D}\left|n_{D}\right| \underset{k}{\operatorname{vol}}(D) \in[0, \infty] .
$$

The boundary of a definable current is again a definable current. It follows that definable currents are locally integral currents in the sense of [13]. In particular, they are locally normal currents, i.e. the mass and the mass of the boundary are finite on compact sets.

If $T \in \mathcal{D}_{k}\left(\mathbb{R}^{n}\right)$ and $A \subset \mathbb{R}^{n}$ are definable, then the current $T\llcorner A$ defined by

$$
T\left\llcorner A=\sum_{D} n_{D}[[D \cap A]],\right.
$$

is again a definable current.

Given a definable $C^{1}$-map $f: \mathbb{R}^{n} \rightarrow \mathbb{R}^{m}$ which is proper on the support of $T$ (i.e. $f^{-1}(K) \cap \operatorname{spt} T$ is compact whenever $K \subset \mathbb{R}^{m}$ is compact), the current $f_{*} T$ with

$$
f_{*} T(\omega):=T\left(f^{*} \omega\right)
$$

is again a definable current, called the image of $T$ under $f$. Note that $f_{*} \circ \partial=$ $\partial \circ f_{*}$.

Given definable currents $S \in \mathcal{D}_{k}\left(\mathbb{R}^{n}\right)$ and $T \in \mathcal{D}_{l}\left(\mathbb{R}^{m}\right)$, their direct product $S \times T \in \mathcal{D}_{k+l}\left(\mathbb{R}^{n} \times \mathbb{R}^{m}\right)$ is defined in the obvious way, i.e. $S \times T$ is defined by integration over the products of the cells of $S$ and $T$, counted with the product of the multiplicities.

Proposition 4.3 (Homotopy formula): Let $T \in \mathcal{D}_{k}\left(\mathbb{R}^{n}\right)$. Suppose

$$
H:[0,1] \times \mathbb{R}^{n} \rightarrow \mathbb{R}^{n}
$$

is a definable $C^{1}$ homotopy of $\mathbb{R}^{n}$ between $f$ and $g$ such that $H^{-1}(K) \cap \operatorname{spt} T$ is compact in $[0,1] \times \mathbb{R}^{n}$ for any compact set $K \subset \mathbb{R}^{n}$. Then

$$
g_{*} T-f_{*} T=\partial H_{*}([0,1] \times T)+H_{*}([0,1] \times \partial T) .
$$

Proof: The assumption implies that the currents

$$
H_{*}(T \times[0,1]) \quad \text { and } \quad H_{*}(\partial T \times[0,1])
$$

are well-defined. The formula follows from

$$
\begin{aligned}
\partial H_{*}([0,1] \times T) & =H_{*} \partial([0,1] \times T) \\
& =H_{*}((\partial[0,1]) \times T)-H_{*}([0,1] \times \partial T) \\
& =\underbrace{H_{*}\left(\delta_{1} \times T\right)}_{g_{*} T}-\underbrace{H_{*}\left(\delta_{0} \times T\right)}_{f_{*} T}-H_{*}([0,1] \times \partial T) .
\end{aligned}
$$


For completeness, we prove a very special case of the constancy theorem ([13], 4.1.7):

Proposition 4.4 (Constancy theorem): If a definable current $T \in \mathcal{D}_{n}\left(\mathbb{R}^{n}\right)$ satisfies $\partial T=0$ and has compact support, then it vanishes.

Proof: Choose a $C^{1}$ cell decomposition of $\mathbb{R}^{n}$ such that $T=\sum_{D} n_{D}[[D]]$. The $n$-cells above an $n-1$-cell $D^{\prime}$ of $\mathbb{R}^{n-1}$ are given by open bands

$$
D_{i}:=\left\{(x, y) \in D^{\prime} \times \mathbb{R}: \xi_{D^{\prime}, i}(x)<y<\xi_{D^{\prime}, i+1}(x)\right\}, \quad i=0, \ldots, l\left(D^{\prime}\right),
$$

where $\xi_{D^{\prime}, 1}<\cdots<\xi_{D^{\prime}, l\left(D^{\prime}\right)}$ are definable $C^{1}$ functions on $D^{\prime}$ and $\xi_{D^{\prime}, 0}=$ $-\infty, \xi_{D^{\prime}, l\left(D^{\prime}\right)+1}=\infty$.

The boundary of $\left[\left[D_{i}\right]\right]$ is given by integration over the graph of $\xi_{D^{\prime}, i+1}$ minus integration over the graph of $\xi_{D^{\prime}, i}$ plus some components lying above the boundary of $D^{\prime}$. These boundaries cancel out only if $n_{D_{i}}=n_{D_{i+1}}$ for $i=0, \ldots, l\left(D^{\prime}\right)-1$. Since the support of $T$ is compact, $n_{D_{0}}=0$ and hence $n_{D_{i}}=0$ for $i=0,1, \ldots, l\left(D^{\prime}\right)$. The same argument works above each $n-1$-cell $D^{\prime}$ of $\mathbb{R}^{n-1}$ and shows that $n_{D}=0$ for all $n$-dimensional cells $D$, i.e. $T=0$.

4.2. Slicing Definable CurRents. The next proposition is contained in [20], [21]. To be precise, it is stated with definable replaced by subanalytic. However, the proof only relies on properties of subanalytic sets which also hold in general o-minimal structures.

Proposition 4.5: Let $T \in \mathcal{D}_{k}\left(\mathbb{R}^{n}\right)$ be a definable current and $f: \mathbb{R}^{n} \rightarrow \mathbb{R}^{l}$ a definable map. Then for all $y \in \mathbb{R}^{l}$ such that $\operatorname{dim} f^{-1}(y) \cap \operatorname{spt} T \leq k-l$ and $\operatorname{dim} f^{-1}(y) \cap \operatorname{spt} \partial T \leq k-l-1$ there exists a definable current $\langle T, f, y\rangle \in$ $\mathcal{D}_{k-l}\left(\mathbb{R}^{n}\right)$, called slice of $T$, with the following properties:

(1) $\operatorname{spt}\langle T, f, y\rangle \subset \operatorname{spt} T \cap f^{-1}(y)$;

(2) $\partial\langle T, f, y\rangle=(-1)^{l}\langle\partial T, f, y\rangle$;

(3) if $g: \mathbb{R}^{n} \rightarrow \mathbb{R}^{m}$ is a definable function which is proper on the support of $T$, and $f: \mathbb{R}^{m} \rightarrow \mathbb{R}^{l}$ any definable function, then

$$
g_{*}\langle T, f \circ g, y\rangle=\left\langle g_{*} T, f, y\right\rangle
$$

whenever $y \in \mathbb{R}^{l}$ and

$\operatorname{dim}(f \circ g)^{-1}(y) \cap \operatorname{spt} T \leq k-l \quad$ and $\quad \operatorname{dim}(f \circ g)^{-1}(y) \cap \operatorname{spt} \partial T \leq k-l-1 ;$

(4) if $f^{\prime}: \mathbb{R}^{n} \rightarrow \mathbb{R}^{l^{\prime}}$ is a definable function, then

$$
\left\langle\langle T, f, y\rangle, f^{\prime}, y^{\prime}\right\rangle=\left\langle T,\left(f, f^{\prime}\right),\left(y, y^{\prime}\right)\right\rangle
$$


for all $y \in \mathbb{R}^{l}, y^{\prime} \in \mathbb{R}^{l^{\prime}}$ such that the following dimension restrictions hold: $\operatorname{dim} f^{-1}(y) \cap \operatorname{spt} T \leq k-l, \operatorname{dim} f^{-1}(y) \cap \operatorname{spt} \partial T \leq k-l-1$, $\operatorname{dim}\left(f^{\prime}\right)^{-1}\left(y^{\prime}\right) \cap \operatorname{spt} T \leq k-l^{\prime}, \operatorname{dim}\left(f^{\prime}\right)^{-1}\left(y^{\prime}\right) \cap \operatorname{spt} \partial T \leq k-l^{\prime}-1$, $\operatorname{dim} f^{-1}(y) \cap\left(f^{\prime}\right)^{-1}\left(y^{\prime}\right) \cap \operatorname{spt} T \leq k-l-l^{\prime}, \operatorname{dim} f^{-1}(y) \cap\left(f^{\prime}\right)^{-1}\left(y^{\prime}\right) \cap \operatorname{spt} \partial T \leq$ $k-l-l^{\prime}-1$;

(5) if $g: \mathbb{R}^{l} \rightarrow \mathbb{R}^{l}$ is a definable diffeomorphism, then

$$
\langle T, g \circ f, y\rangle=\varepsilon\left\langle T, f, g^{-1}(y)\right\rangle
$$

whenever $y \in \mathbb{R}^{l}$ with

$\operatorname{dim}(g \circ f)^{-1}(y) \cap \operatorname{spt} T \leq k-l \quad$ and $\quad \operatorname{dim}(g \circ f)^{-1}(y) \cap \operatorname{spt} \partial T \leq k-l-1$.

Here $\varepsilon=1$ if $g$ is orientation preserving, and -1 else.

Note that the conditions on the dimensions are satisfied for almost all $y \in \mathbb{R}^{l}$. The above statement, but with the condition on the dimensions replaced by for almost all $y \in \mathbb{R}^{l}$, is well-known for any (not necessarily definable) normal current $T$ (compare with [13], 4.3). In the proof of existence and uniqueness of normal cycles, we will only use this weaker version of the above proposition. Only in the construction of the normal cycles associated to projections and convolutions of constructible functions, we will have to slice at special values and then we verify that the condition on the dimensions is satisfied.

For our purposes, the most important case is where the function $f$ is the orthogonal projection $\pi_{W}$ on a subspace $W$ with $\operatorname{dim} W=\operatorname{dim} T$. In this case, we find a $C^{2}$-cell decomposition of spt $T$ compatible with $\pi_{W}$. If $D^{\prime}$ is a cell of highest dimension in $W$, then $\pi_{W}^{-1}\left(D^{\prime}\right) \cap \operatorname{spt} T$ is a union of graphs on $D^{\prime}$. It follows that for $y \in D^{\prime}$, the intersection $\pi_{W}^{-1}(y) \cap \operatorname{spt} T$ is a finite union of points and the slice $\left\langle T, \pi_{W}, y\right\rangle$ (which is 0-dimensional) is the sum of the corresponding Dirac measures, counted with multiplicities according to the multiplicities of the cells of spt $T$.

4.3. Support Functions of Legendrian CyCles. We fix the following notation. The canonical projections from $V \oplus V$ to $V$ are denoted $\pi_{1}$ and $\pi_{2}$, the canonical embeddings from $V$ into $V \oplus V$ are denoted $\tau_{1}, \tau_{2}$. We define maps $m: \mathbb{R} \oplus V \oplus V \rightarrow V \oplus V,(\lambda, x, y) \mapsto(x, \lambda y)$ and $m_{\lambda}: V \oplus V \rightarrow$ $V \oplus V, m_{\lambda}(x, y):=m(\lambda, x, y)$. Note that $m_{0}=\tau_{1} \circ \pi_{1}$. The scalar product is denoted by $u: V \oplus V \rightarrow \mathbb{R},(x, y) \mapsto\langle x, y\rangle$.

The canonical 1-form $\alpha$ on $V \oplus V$ is defined by $\alpha(v)=\left\langle y,\left(\pi_{1}\right)_{*} v\right\rangle$ for $v \in$ $T_{(x, y)}(V \oplus V)$. We will not distinguish notationally between $\alpha$ and its restriction to $S V$, making the latter space into a contact manifold. 
Definition 4.6: A Legendrian current is a current $T \in \mathcal{D}_{n-1}(V \oplus V)$ such that $\operatorname{spt} T$ is contained in $S V$ and such that

$$
T\llcorner\alpha=0 .
$$

Definition 4.7: We call a current $S \in \mathcal{D}_{n}(V \oplus V)$ conical if

$$
\left(m_{\lambda}\right)_{*} S=S
$$

for $\lambda>0 . S$ is called Lagrangian if, with $\omega:=-d \alpha$ denoting the symplectic form on $V \oplus V$,

$$
S\llcorner\omega=0 .
$$

We recall that a linear subspace $W$ of $V \oplus V$ is called isotropic if $\left.\omega\right|_{W}=0$. Then $\operatorname{dim} W \leq n$ and $W$ is called Lagrangian if $\operatorname{dim} W=n$.

Proposition 4.8:

(1) If $T$ is a Legendrian cycle, then $T\llcorner\omega=0$.

(2) If $S$ is a conical, definable Lagrangian current on $V \oplus V$, then $S\llcorner\alpha=0$.

(3) There is a one-to-one correspondence between compactly supported definable Legendrian cycles $T$ and definable, conical Lagrangian cycles $S$ on $V \oplus V$ such that $\pi_{1}(\operatorname{spt} S)$ is compact.

Proof: (Compare with [16])

(1) Let $\phi$ be an $n-3$-form on $V \oplus V$. Since $\partial T=0$ and $T\llcorner\alpha=0$, we obtain $T\llcorner\omega(\phi)=-T(d \alpha \wedge \phi)=T(\alpha \wedge d \phi)=0$.

(2) If $D$ is an $n$-dimensional cell in the support of $S$ and $(x, y) \in D$, then $T_{(x, y)} D$ is Lagrangian. Let $v$ be the gradient vector field of the function $(x, y) \mapsto \frac{1}{2}\|y\|^{2}$. Since $S$ is conical, $v \in T_{(x, y)} D$ and thus $\left.\alpha\right|_{T_{(x, y)} D}=$ $-v\lrcorner\left.\omega\right|_{T_{(x, y)} D}=0$.

(3) We write $[0, \infty)$ not only for the interval, but also for the 1-current defined by integration over it. Given a compactly supported definable Legendrian cycle $T$,

$$
\partial m_{*}([0, \infty) \times T)=-m_{*}\left(\delta_{0} \times T\right)=-\left(\tau_{1}\right)_{*}\left(\pi_{1}\right)_{*} T .
$$

The current $\left(\pi_{1}\right)_{*} T$ is a compactly supported definable $n$ - 1-cycle in $V$ and can be filled by a compactly supported definable $n$-current $U$, i.e. $\partial U=\left(\pi_{1}\right)_{*} T$. Then

$$
S:=m_{*}([0, \infty) \times T)+\left(\tau_{1}\right)_{*} U
$$


is a conical Lagrangian cycle and $\pi_{1}(\operatorname{spt} S) \subset \pi_{1}(\operatorname{spt} T) \cup \operatorname{spt} U$ is compact.

Now suppose that $S$ is a conical Lagrangian cycle with $\pi_{1}(\operatorname{spt} S)$ compact. We can assume that $S$ is given by integration over oriented conical cells and define the current $T$ by intersecting $S$ with $S V$ (i.e. by taking intersections of the conical cells of $S$ with $S V$, with the same multiplicities). Then $T$ is a compactly supported Legendrian cycle.

It can be checked that the operations $T \mapsto S, S \mapsto T$ are inverse to each other, finishing the proof.

Definition 4.9: $\quad$ Let $T \in \mathcal{D}_{n-1}(V \oplus V)$ be a compactly supported, definable Legendrian cycle and $S$ the associated Lagrangian cycle. The support function of $T$ is the (almost everywhere defined) function $h_{T}: V \mapsto \mathbb{Z}[R]$ with

$$
h_{T}(y):=u_{*}\left\langle S, \pi_{2}, y\right\rangle .
$$

Since $S$ is conical, the support function of a Legendrian cycle $T$ is (almost everywhere) homogeneous in the sense of $2.12,(2)$ and can thus be identified with a function on $S(V)$.

\section{Lipschitz continuity of support functions}

Theorem 5.1: Let $T \in \mathcal{D}_{n-1}(V \oplus V)$ be a definable Legendrian cycle with compact support. Then $h_{T}$ can be extended to a definable Lipschitz continuous function $V \rightarrow \mathbb{Z}[\mathbb{R}]$ (with respect to $\mathbf{F}$ ).

\section{Proof:}

SteP 1: Let $S$ be the definable, conical Lagrangian cycle associated to $T$. Suppose that $\operatorname{spt} S \subset B(0, R) \times V$.

Fix a definable $C^{1}$-cell decomposition of $V \oplus V$, compatible with $\operatorname{spt} S$ and $\pi_{2}$ (compare with Theorem 2.3). By reverse induction we can also achieve that the boundary of a cell in $V$ is a union of cells.

Let $D \subset \operatorname{spt} S$ be an $n$-dimensional cell and $(x, y) \in D$. Given $v \in T_{(x, y)} D$, the Legendrian condition implies that $\left\langle y, d \pi_{1}(v)\right\rangle=0$. Therefore $d u(v)=$ $\left\langle x, d \pi_{2}(v)\right\rangle$, which implies that

$$
d\left(\pi_{2}, u\right)(v)=\left(d \pi_{2}(v),\left\langle x, d \pi_{2}(v)\right\rangle\right) .
$$


Suppose first that the rank of $\left.\pi_{2}\right|_{D}$ is $n$. Then $D^{\prime}:=\pi_{2}(D) \subset V$ is an $n$ dimensional cell and $D$ is the graph of a definable, $C^{1}$-smooth function $g: D^{\prime} \rightarrow V$. With $f(y):=\langle g(y), y\rangle$ for $y \in D^{\prime}$ we get $\left(\pi_{2}, u\right)(D)=\operatorname{graph} f$.

From Equation (1) we deduce that $\operatorname{grad} f(y)=g(y)$ for all $y \in D^{\prime}$. Since $(g(y), y) \in \operatorname{spt} S \subset B(0, R) \times V$, the norm of the gradient of $f$ is bounded by $R$, which implies that $f$ is locally $R$-Lipschitz on $D^{\prime}$.

If the rank of $\left.\pi_{2}\right|_{D}$ is less than $n$, then Equation (1) implies that also the rank of $\left.\left(\pi_{2}, u\right)\right|_{D}$ is less than $n$ and thus $\left(\pi_{2}, u\right)_{*}[[D]]=0$.

We obtain that $G:=\left(\pi_{2}, u\right)_{*} S$ is given by integration over finitely many (say $M$ ) cells of $V \times \mathbb{R}$ which are graphs of locally $R$-Lipschitz functions on $n$-dimensional cells in $V$. In particular, $G$ has no vertical components.

Note further that, with $\pi_{z}: V \times \mathbb{R} \rightarrow \mathbb{R},(y, z) \mapsto z$ and $\pi_{y}: V \times \mathbb{R} \rightarrow V$, $(y, z) \mapsto y$, we get for almost all $y \in V$

$$
\begin{aligned}
\left(\pi_{z}\right)_{*}\left\langle G, \pi_{y}, y\right\rangle & =\left(\pi_{z}\right)_{*}\left\langle\left(\pi_{2}, u\right)_{*} S, \pi_{y}, y\right\rangle \\
& =\underbrace{\left(\pi_{z}\right)_{*} \circ\left(\pi_{2}, u\right)_{*}}_{=u_{*}}\langle S, \underbrace{\pi_{y} \circ\left(\pi_{2}, u\right)}_{=\pi_{2}}, y\rangle \\
& =h(y),
\end{aligned}
$$

i.e. $G$ can be considered as "graph" of $h$.

SteP 2: Let $y$ belong to an $n-1$-dimensional cell. Then $h$ is continuous at $y$. Lemma 5.2: Let $D \subset V$ be a $k$-cell and let $f: D \rightarrow \mathbb{R}$ be a bounded and definable $C^{1}$-function. Then there exists a definable $C^{2}$-cell decomposition of $\partial D$ such that for each cell $D^{\prime}$ of dimension $k-1$ there exists a unique continuous extension of $f$ on $D \cup D^{\prime}$.

Proof: This is a standard argument, a sketch of which will be given. We fix cell decompositions of the boundary of the graph of $f$ (which is a bounded, definable, $k$-1-dimensional subset of $V \times \mathbb{R}$ ) and of the boundary of $D$ which are compatible with the projection to $V$. Above a $k-1$-dimensional cell $D^{\prime} \subset \partial D$, there can only be finitely many $k-1$-dimensional cells. Since $D$ is locally connected, there is exactly one such cell and the result follows.

By the Lemma, we find a refinement of the cell decomposition in such a way that each of the functions $f: D^{\prime} \rightarrow \mathbb{R}$ can be continuously extended to $n-1$-cells in the boundary of $D^{\prime}$.

Let $D^{\prime \prime}$ be a cell of $V$ of dimension $n-1$. Let $D_{1}^{\prime}$ and $D_{2}^{\prime}$ be the two $n$-cells of $V$ containing $D^{\prime \prime}$ in their boundary. Note that the induced orientations on $D^{\prime \prime}$ do not coincide. 
By Step 1, there are representations of the form

$$
\begin{array}{ll}
h(y)=\sum_{i=1}^{k} a_{i} \delta_{f_{i}(y)} \quad \forall y \in D_{1}^{\prime} \\
h(y)=\sum_{j=1}^{l} b_{j} \delta_{g_{j}(y)} \quad \forall y \in D_{2}^{\prime},
\end{array}
$$

with locally $R$-Lipschitz continuous functions $f$ and $g$.

By construction, the functions $f_{i}$ (resp. $g_{j}$ ) extend by continuity to $D_{1}^{\prime} \cup D^{\prime \prime}$ (resp. $D_{2}^{\prime} \cup D^{\prime \prime}$ ). Let $r: D^{\prime \prime} \rightarrow \mathbb{R}$ be the restriction of such a function to $D^{\prime \prime}$.

Let $I_{r} \subset\{1, \ldots, k\}$ be the set of indices $i$ such that $\left.f_{i}\right|_{D^{\prime \prime}}=r$ and $J_{r} \subset$ $\{1, \ldots, l\}$ be the set of indices $j$ such that $\left.g_{j}\right|_{D^{\prime \prime}}=r$.

Since each $i$ belongs to exactly one $I_{r}$ and each $j$ belongs to exactly one $J_{r}$, and since $G$ has no vertical components, we get

$$
\begin{aligned}
G\left\llcorner\pi_{y}^{-1}\left(D_{1}^{\prime} \cup D_{2}^{\prime}\right)\right. & = \\
& \sum_{r}\left(\sum_{i \in I_{r}} a_{i}\left[\left[\operatorname{graph} f_{i}: D_{1}^{\prime} \rightarrow \mathbb{R}\right]\right]+\sum_{j \in J_{r}} b_{j}\left[\left[\operatorname{graph} g_{j}: D_{2}^{\prime} \rightarrow \mathbb{R}\right]\right]\right)
\end{aligned}
$$

and

$$
\begin{aligned}
\partial G\left\llcorner D^{\prime \prime}\right. & =\sum_{r}\left(\sum_{i \in I_{r}} a_{i} \partial\left[\left[\operatorname{graph} f_{i}: D_{1}^{\prime} \rightarrow \mathbb{R}\right]\right]+\sum_{j \in J_{r}} b_{j} \partial\left[\left[\operatorname{graph} g_{j}: D_{2}^{\prime} \rightarrow \mathbb{R}\right]\right]\right) \\
& =\sum_{r}\left(\sum_{i \in I_{r}} a_{i}\left[\left[\operatorname{graph} f_{i}: D^{\prime \prime} \rightarrow \mathbb{R}\right]\right]-\sum_{j \in J_{r}} b_{j}\left[\left[\operatorname{graph} g_{j}: D^{\prime \prime} \rightarrow \mathbb{R}\right]\right]\right) \\
& =\sum_{r}\left(\sum_{i \in I_{r}} a_{i}-\sum_{j \in J_{r}} b_{j}\right)\left[\left[\operatorname{graph} r: D^{\prime \prime} \rightarrow \mathbb{R}\right]\right] .
\end{aligned}
$$

On the other hand, $\partial G=\left(\pi_{2}, u\right)_{*} \partial S=0$ and thus $\sum_{i \in I_{r}} a_{i}=\sum_{j \in J_{r}} b_{j}$ for all $r$.

Let $y \in D^{\prime \prime}$ and $\epsilon>0$. Since $f_{i}$ and $g_{j}$ can be continuously extended to $D^{\prime \prime}$, we get for all $y_{1} \in D_{1}^{\prime}$ and $y_{2} \in D_{2}^{\prime}$ sufficiently close to $y$ that $\left|f_{i}\left(y_{1}\right)-f_{i}(y)\right| \leq \epsilon$ and $\left|g_{j}\left(y_{2}\right)-g_{j}(y)\right| \leq \epsilon$.

From

$$
\sum_{i \in I_{r}} a_{i} \delta_{f_{i}(y)}=\sum_{i \in I_{r}} a_{i} \delta_{r(y)}=\sum_{j \in J_{r}} b_{j} \delta_{r(y)}=\sum_{j \in J_{r}} b_{j} \delta_{g_{j}(y)}
$$


we deduce that

$$
\begin{aligned}
\mathcal{F}\left(h\left(y_{1}\right)\right. & \left.-h\left(y_{2}\right)\right) \\
& =\mathcal{F}\left(\sum_{r}\left(\sum_{i \in I_{r}} a_{i} \delta_{f_{i}\left(y_{1}\right)}-\sum_{j \in J_{r}} b_{j} \delta_{g_{j}\left(y_{2}\right)}\right)\right) \\
& \leq \mathcal{F}\left(\sum_{r} \sum_{i \in I_{r}} a_{i}\left(\delta_{f_{i}\left(y_{1}\right)}-\delta_{f_{i}(y)}\right)\right)+\mathcal{F}\left(\sum_{r} \sum_{j \in J_{r}} b_{j}\left(\delta_{g_{j}\left(y_{2}\right)}-\delta_{g_{j}(y)}\right)\right) \\
& \leq\left(\sum_{i=1}^{k}\left|a_{i}\right|+\sum_{j=1}^{l}\left|b_{j}\right|\right) \epsilon .
\end{aligned}
$$

This proves the claim.

SteP 3: Let $y_{1}, y_{2} \in V$ be both contained in $n$-dimensional cells. For sufficiently small $\epsilon>0$, each point $y_{1}^{\prime}$ with $\left\|y_{1}^{\prime}-y_{1}\right\| \leq \epsilon$ satisfies $\mathcal{F}\left(h\left(y_{1}^{\prime}\right)-h\left(y_{1}\right)\right) \leq$ $M R\left\|y_{1}^{\prime}-y_{1}\right\|$ and similarly for $y_{2}^{\prime}$ (see Step 1). With a random choice of $y_{1}^{\prime} \in B\left(y_{1}, \epsilon\right)$ and $y_{2}^{\prime} \in B\left(y_{2}, \epsilon\right)$, the line between $y_{1}^{\prime}$ and $y_{2}^{\prime}$ crosses finitely many $n$-1-dimensional cells and stays in the union of the $n$-dimensional cells otherwise.

By Step 1, the restriction of $h$ to this line is locally $M R$-Lipschitz except for a finite number of points. In these points, $h$ is continuous by Step 2. We deduce that $h$ is $M R$-Lipschitz on this line, and it follows that

$$
\begin{aligned}
\mathcal{F}\left(h\left(y_{1}\right)-h\left(y_{2}\right)\right) & \leq \mathcal{F}\left(h\left(y_{1}\right)-h\left(y_{1}^{\prime}\right)\right)+\mathcal{F}\left(h\left(y_{1}^{\prime}\right)-h\left(y_{2}^{\prime}\right)\right)+\mathcal{F}\left(h\left(y_{2}^{\prime}\right)-h\left(y_{2}\right)\right) \\
& \leq 2 M R \epsilon+M R\left\|y_{1}^{\prime}-y_{2}^{\prime}\right\| \\
& \leq 4 M R \epsilon+M R\left\|y_{1}-y_{2}\right\| .
\end{aligned}
$$

Since $\epsilon$ can be chosen arbitrarily small, we obtain $\mathcal{F}\left(h\left(y_{1}\right)-h\left(y_{2}\right)\right) \leq$ $M R\left\|y_{1}-y_{2}\right\|$. Since the union of all $n$-dimensional cells is dense in $V, h$ can be extended to an $M R$-Lipschitz continuous, definable function on $V$.

\section{Construction of the normal cycle}

We recall that $V$ denotes an oriented, $n$-dimensional Euclidean vector space.

Definition 6.1: Let $\phi: V \rightarrow \mathbb{Z}$ be a constructible function with compact support. A compactly supported, definable Legendrian cycle $T \in \mathcal{D}_{n-1}(V \oplus V)$ is called normal cycle of $\phi$ if $h_{T}=h_{\phi}$ almost everywhere.

Remark: The normal cycle of $\phi$ depends on the orientation of $V$. Indeed, changing the orientation of $V$ does not alter $h_{\phi}$, but $\left\langle T, \pi_{2}, y\right\rangle$ depends on the 
orientation of the target space $V$. Therefore, the normal cycle of $\phi$ with respect to the reversed orientation is minus the normal cycle of $\phi$ with respect to the given one.

Theorem 6.2 (Existence and uniqueness of the normal cycle): Each compactly supported constructible function $\phi$ admits a unique normal cycle. Conversely, each compactly supported definable Legendrian cycle is the normal cycle of a unique constructible function with compact support.

The first part of this theorem was discovered by Fu ([15]) using deep methods from Geometric Measure Theory. The proof we will give below uses only Lipschitz continuity of support functions and basic properties of constructible functions and definable currents.

We will use the following notation. The normal cycle of a compactly supported constructible function will be denoted by $T_{\phi}$. The corresponding conical Lagrangian cycle will be denoted by $S_{\phi}$. Given a compactly supported Legendrian cycle $T$ (or a conical Lagrangian cycle $S$ with $\pi_{1}$ (spt $S$ ) compact), we denote by $\phi_{T}$ (or $\phi_{S}$ ) the unique compactly supported constructible function with normal cycle $T$.

Proof: The proof of the second part was already given. Indeed, if $T$ is a compactly supported definable Legendrian cycle, then $h=h_{T}$ is definable, homogeneous and Lipschitz (Theorem 5.1). Theorem 3.1 implies that there is a unique constructible function $\phi$ with compact support such that $h_{\phi}=h$.

Conversely, let $\phi$ be constructible with compact support. By Theorem 3.1, $h=h_{\phi}$ is definable, homogeneous and $L$-Lipschitz for some $L>1$ (with respect to $\mathbf{F}$ ). We have to show that there exists a unique compactly supported, definable Legendrian cycle $T$ with $h_{T}=h$.

Existence:

Lemma 6.3: Let $D \subset V$ be a $C^{2}$-cell and $f \in C^{2}(D)$. Suppose that $D$ is conical and $f$ is homogeneous, i.e. $\lambda D=D$ and $f(\lambda y)=\lambda f(y)$ for all $\lambda>0, y \in D$. Then

$$
\Gamma(D, f):=\left\{(x, y) \in V \oplus V: y \in D,\langle x, v\rangle=v(f) \forall v \in T_{y} D\right\}
$$

is a conical Lagrangian submanifold of $V \oplus V$.

Proof: Since $D$ is conical, $y \in T_{y} D$ for all $y \in D$. By homogeneity of $f$, $\langle x, y\rangle=f(y)$ for all $(x, y) \in \Gamma(D, f)$. 
Let $(x(t), y(t))$ be a differentiable curve in $\Gamma(D, f)$ with $(x(0), y(0))=(x, y)$. Then $v:=y^{\prime}(0) \in T_{y} D$. We obtain

$$
\left.\frac{d}{d t}\right|_{t=0}\langle x(t), y(t)\rangle=\left.\frac{d}{d t}\right|_{t=0} f(y(t))=v(f) .
$$

On the other hand,

$$
\left.\frac{d}{d t}\right|_{t=0}\langle x(t), y(t)\rangle=\left\langle x^{\prime}(0), y\right\rangle+\underbrace{\left\langle x, y^{\prime}(0)\right\rangle}_{=v(f)} .
$$

Comparing these formulas yields that $\left\langle x^{\prime}(0), y\right\rangle=0$, which shows that $\left.\alpha\right|_{\Gamma(D, f)}=0$. Differentiation yields $\left.d \alpha\right|_{\Gamma(D, f)}=0$, i.e. $\Gamma(D, f)$ is Lagrangian.

It is clear that $\Gamma(D, f)$ is conical.

Lemma 6.4: Let $D, f$ be as in the preceding lemma and suppose that $\|\operatorname{grad} f\| \leq L$. Let $S$ be a definable, conical, $n$-1-dimensional current on $V \oplus V$ with spt $S \subset \overline{\Gamma(D, f)}$, spt $\partial S \subset \partial \Gamma(D, f)$ and $\pi_{1}(\operatorname{spt} S)$ compact.

If $\operatorname{dim} D<n-1$ or $\operatorname{dim} D=n-1$ and $\left(\pi_{2}\right)_{*} S=0$, then there exists a conical, definable $n$-current $S^{\prime}$ with spt $S^{\prime} \subset \overline{\Gamma(D, f)}, \operatorname{spt}\left(\partial S-S^{\prime}\right) \subset \partial \Gamma(D, f)$ and such that $\pi_{1}\left(\operatorname{spt} S^{\prime}\right)$ is contained in the convex hull of $\pi_{1}(\operatorname{spt} S) \cup B(0, L)$.

Proof: For $y \in D$, set $g(y):=\sum_{i=1}^{\operatorname{dim} D} e_{i}(f) e_{i}$, where $e_{1}, \ldots, e_{\operatorname{dim} D}$ is an orthonormal base of $T_{y} D$ (if $\operatorname{dim} D=0$, set $g(y)=0$ ). Clearly $(g(y), y) \in \Gamma(D, f)$ and $\|g(y)\| \leq L$.

Define a homotopy

$$
H:[0,1] \times \Gamma(D, f) \rightarrow \Gamma(D, f),(t,(x, y)) \mapsto(t x+(1-t) g(y), y)
$$

and set $S^{\prime}:=H_{*}([0,1] \times S)$.

By the homotopy formula 4.3 , up to a current with support in $\partial \Gamma(D, f)$,

$$
\partial S^{\prime}=\underbrace{H_{*}\left(\delta_{1} \times S\right)}_{=S}-H_{*}\left(\delta_{0} \times S\right) .
$$

If $\operatorname{dim} D<n-1$, then the second term vanishes since it is an $n-1$-current supported in the $\operatorname{dim} D$-dimensional set $\{(g(y), y): y \in D\}$.

If $\operatorname{dim} D=n-1$ and $\left(\pi_{2}\right)_{*} S=0$, then

$$
H_{*}\left(\delta_{0} \times S\right)=\left(H_{0}\right)_{*} S=(g, \mathrm{id})_{*} \circ\left(\pi_{2}\right)_{*} S=0 .
$$

$\pi_{1}\left(\operatorname{spt} S^{\prime}\right)$ is contained in the convex hull of $\pi_{1}(\operatorname{spt} S) \cup B(0, L)$, in both cases. 
Lemma 6.5: Let $h: V \rightarrow \mathbb{Z}[\mathbb{R}]$ be homogeneous, definable and L-Lipschitz. Then there exist finite $C^{2}$-cell decompositions of $V \oplus V$ and $V$, compatible with $\pi_{2}$, such that

(1) each cell $D \subset V$ is conical and each cell $\tilde{D} \subset V \oplus V$ is conical in the second coordinate;

(2) for each cell $D \subset V$, there exists a finite family $F(D)$ of definable $C^{2}$ functions $f_{1}<f_{2}<\cdots<f_{k}$ and integers $a_{1}, \ldots, a_{k}$ with

$$
h(y)=\sum_{i=1}^{k} a_{i} \delta_{f_{i}(y)} \quad \forall y \in D ;
$$

(3) if $D^{\prime} \subset \partial D, \operatorname{dim} D^{\prime}=\operatorname{dim} D-1$ and $f \in F(D)$, then there exists $f^{\prime} \in$ $F\left(D^{\prime}\right)$ which is the restriction of the continuous extension of $f$ to $D^{\prime}$;

(4) the boundary of each cell is a union of cells;

(5) if $D \subset V$ is a cell and $f \in F(D)$, then $\Gamma(D, f)$ is a union of cells.

Proof: In the first step, we construct a cell decomposition of $V$ with (1)-(4). Since $h$ is homogeneous and definable, we find a conical cell decomposition of $V$ such that $h$ is given above each cell by $h(y)=\sum_{i=1}^{k} a_{i} \delta_{f_{i}(y)}$. For $n$-dimensional cells $D$, we set $F(D)=\left(f_{1}, \ldots, f_{k}\right)$. Using Lemma 5.2 , we can subdivide the $n-1$-skeleton in such a way that all functions $f \in F(D)$ can be continuously extended to cells of dimension $n-1$. For a cell $D^{\prime}$ of dimension $n-1$, we let $F\left(D^{\prime}\right)$ be the set of restrictions of all functions belonging to some $F(D)$ with $\operatorname{dim} D=n$ and $D^{\prime} \subset \partial D$.

Subdividing the $n-2$-skeleton, we can assume that all functions $f \in F(D)$, $\operatorname{dim} D=n-1$, extend continuously to $n-2$-cells. We define $F\left(D^{\prime}\right)$ for $n-2$-cells similarly as above and continue in this way. After $n$ steps, we get a cell decomposition of $V$ with (1)-(4).

Note that any subdivision of this cell decomposition also satisfies (1)-(4) (we let $F\left(D^{\prime}\right)$ be the set of restrictions of functions from $F(D)$, where $D$ is the unique cell of the original decomposition containing $\left.D^{\prime}\right)$.

In the second step, we construct a cell decomposition of $V \oplus V$ which is $\pi_{2}$-compatible with some subdivision of the given cell decomposition and which satisfies (4) and (5). We choose a cell decomposition of $V \oplus V$ such that $\Gamma(D, f)$ is a union of cells for each $D$ of dimension $n$ and $f \in F(D)$. By subdividing, we can achieve that the boundary of each cell is a cell. By subdividing again, we achieve that the sets $\Gamma(D, f), f \in F(D)$ with $\operatorname{dim} D=n-1$ are unions of cells. Continuing in this way, we obtain $\pi_{2}$-compatible cell decompositions with (1)-(5). 
We fix cell decompositions as in Lemma 6.5 and set

$$
\mathcal{Y}_{\leq k}:=\bigcup_{\operatorname{dim} D \leq k, f \in F(D)} \Gamma(D, f)
$$

Lemma 6.6: Let $D \subset V$ be a cell and $f \in F(D)$. Then

$$
\partial \Gamma(D, f) \subset \mathcal{Y}_{\leq \operatorname{dim} D-1} \cup M
$$

where $M$ is a subset of dimension $<n-1$.

Proof: Let $\tilde{D}_{1} \subset \partial \Gamma(D, f)$ be an $n-1$-cell and $D_{1}:=\pi_{2}\left(\tilde{D}_{1}\right)$. Then $D_{1} \subset \partial D$, in particular $\operatorname{dim} D_{1} \leq \operatorname{dim} D-1$. By Lemma 6.3 and Stokes's theorem, $\alpha$ vanishes on $\tilde{D}_{1}$.

Let $(x, y) \in \tilde{D}_{1}$. Then there exists a sequence $\left(x_{i}, y_{i}\right) \in \Gamma(D, f)$ converging to $(x, y)$. As was remarked above, $\left\langle x_{i}, y_{i}\right\rangle=f\left(y_{i}\right)$. By continuity, $\langle x, y\rangle=f(y)$.

Consider a differentiable curve $\gamma(t)=(x(t), y(t))$ in $\tilde{D}_{1}$ with $(x, y)=$ $(x(0), y(0))$ and set $v:=y^{\prime}(0)$. Then

$$
v(f)=\left.\frac{d}{d t}\right|_{t=0} f(y(t))=\left.\frac{d}{d t}\right|_{t=0}\langle x(t), y(t)\rangle=\langle x, v\rangle+\underbrace{\left\langle x^{\prime}(0), y(0)\right\rangle}_{=\alpha\left(\gamma^{\prime}(0)\right)=0} .
$$

Since $\pi_{2}: \tilde{D}_{1} \rightarrow D_{1}$ is submersive, it follows that $(x, y) \in \Gamma\left(D_{1}, f\right)$.

Now we can complete the construction of the normal cycle.

We define a sequence of currents $S_{k}, k=0,1, \ldots, n$ such that

- $S_{k}$ is a conical, definable Lagrangian current with $\pi_{1}\left(\operatorname{spt} S_{k}\right) \subset B(0, L)$;

- $u_{*}\left\langle S_{k}, \pi_{2}, y\right\rangle=h(y)$ for almost all $y \in V$;

- $\operatorname{spt} \partial S_{k} \subset \overline{\mathcal{Y}}_{\leq n-k-1}$.

For a cell $D$ of dimension $n,\left.h\right|_{D}$ is given as a finite combination

$$
\left.h\right|_{D}=\sum_{i=1}^{k(D)} a_{i}^{D} \delta_{f_{i}^{D}}
$$

with functions $f_{1}^{D}<\cdots<f_{k(D)}^{D}$ from $F(D)$.

We set

$$
S_{0}:=\sum_{\operatorname{dim} D=n} \sum_{i=1}^{k(D)} a_{i}^{D}\left[\left[\Gamma\left(D, f_{i}^{D}\right)\right]\right]
$$

From Lemma 6.3 we deduce that $S_{0}$ is a definable, conical, Lagrangian current. 
Moreover, $\pi_{1}$ (spt $\left.S_{0}\right) \subset B(0, L)$ by Lemma 3.4 and

$$
\begin{aligned}
u_{*}\left\langle S_{0}, \pi_{2}, y\right\rangle & =\sum_{i=1}^{k(D)} a_{i}^{D} u_{*}\left\langle\left[\left[\Gamma\left(D, f_{i}^{D}\right)\right]\right], \pi_{2}, y\right\rangle \\
& =\sum_{i=1}^{k(D)} a_{i}^{D} \delta_{\left\langle\operatorname{grad} f_{i}^{D}(y), y\right\rangle} \\
& =\sum_{i=1}^{k(D)} a_{i}^{D} \delta_{f_{i}^{D}(y)} \\
& =h(y)
\end{aligned}
$$

for $y \in D, \operatorname{dim} D=n$. This means that $u_{*}\left\langle S_{0}, \pi_{2}, y\right\rangle=h(y)$ for almost all $y \in V$.

From Lemma 6.6 we see that $\partial S_{0}$ is a cycle supported in $\overline{\mathcal{Y}}_{\leq n-1}$.

Let $D$ be an $n$-1-cell, $f \in F(D)$ and $D_{1}, D_{2}$ be the $n$-cells neighboring $D$. Then

$$
\left(\partial\left[\left[D_{1}\right]\right]+\partial\left[\left[D_{2}\right]\right]\right)\llcorner D=0 .
$$

Suppose $h(y)=\sum_{i=1}^{k} a_{i} \delta_{f_{i}}$ on $D_{1}$. Let $s_{1}$ be the sum of those $a_{i}$ for which $\left.f_{i}\right|_{D}=f$. We define $s_{2}$ in a similar way. From the continuity of the support function, we obtain $s_{1}=s_{2}$. Indeed, by (2) and (3), both $s_{1}$ and $s_{2}$ equal the coefficient of $\delta_{f}$ in $h \mid D$.

For each function $f_{i}$ with $\left.f_{i}\right|_{D}=f$ (and only for those), we get as in the proof of Lemma 6.6 that

$$
\partial \Gamma\left(D_{1}, f_{i}\right) \cap \pi_{2}^{-1} D \subset \Gamma(D, f)
$$

and

$$
\left(\pi_{2}\right)_{*}\left(\partial\left[\left[\Gamma\left(D_{1}, f_{i}\right)\right]\right]\left\llcorner\pi_{2}^{-1} D\right)=\left(\pi_{2}\right)_{*} \partial\left[\left[\Gamma\left(D_{1}, f_{i}\right)\right]\right]\left\llcorner D=\partial\left[\left[D_{1}\right]\right]\llcorner D .\right.\right.
$$

In the same way, if $h(y)=\sum_{i=1}^{k^{\prime}} a_{i}^{\prime} \delta_{f_{i}^{\prime}}$ on $D_{2}$ and if $\left.f_{i}^{\prime}\right|_{D}=f$, then

$$
\left(\pi_{2}\right)_{*}\left(\partial\left[\left[\Gamma\left(D_{2}, f_{i}^{\prime}\right)\right]\right]\left\llcorner\pi_{2}^{-1} D\right)=\partial\left[\left[D_{2}\right]\right]\llcorner D .\right.
$$

We deduce that

$$
\left(\pi_{2}\right)_{*}\left(\partial S_{0}\llcorner\Gamma(D, f))=s_{1} \partial\left[\left[D_{1}\right]\right]\left\llcorner D+s_{2} \partial\left[\left[D_{2}\right]\right]\llcorner D=0 .\right.\right.
$$

We apply Lemma 6.4 to the currents $\left(\partial S_{0}\right)\llcorner\Gamma(D, f)$ (where $D$ runs over all $n$-1-dimensional cells and $f \in F(D))$ to deduce that there exists a conical, 
definable, Lagrangian current $S_{0}^{\prime}$ with $\pi_{1}\left(\operatorname{spt} S_{0}^{\prime}\right) \subset B(0, L)$, spt $S_{0}^{\prime} \subset \overline{\mathcal{Y}}_{n-1}$ and $\operatorname{spt}\left(\partial S_{0}-\partial S_{0}^{\prime}\right) \subset \overline{\mathcal{Y}}_{\leq n-2}$. Hence $S_{1}:=S_{0}-S_{0}^{\prime}$ satisfies all conditions.

Suppose $S_{k}, 0<k<n$ is already defined. Then $\partial S_{k}$ is an $n-1$ cycle with support in $\overline{\mathcal{Y}}_{\leq n-k-1}$. Applying Lemma 6.4 yields a conical, definable, Lagrangian current $S_{k}^{\prime}$ with $\pi_{1}\left(\operatorname{spt} S_{k}^{\prime}\right) \subset B(0, L)$, spt $S_{k}^{\prime} \subset \overline{\mathcal{Y}}_{n-k-1}$ and $\operatorname{spt}\left(\partial S_{k}-\partial S_{k}^{\prime}\right) \subset \overline{\mathcal{Y}}_{\leq n-k-2}$. Hence $S_{k+1}:=S_{k}-S_{k}^{\prime}$ satisfies all conditions.

In particular, $S:=S_{n}$ is a conical, definable Lagrangian cycle such that $h_{S}(y)=h(y)$ for almost all $y \in V$ and $\pi_{1}$ (spt $\left.S\right) \subset B(0, L)$. Let $T$ be the associated Legendrian cycle. Then $T$ is compactly supported, definable and $h_{T}(y)=h_{S}(y)=h(y)$ for almost all $y \in V$.

Uniqueness: It suffices to show that $h_{S}=0$ implies $S=0$ for compactly supported, definable conical Lagrangian cycles $S$.

ClaIm: $h_{S}=0$ implies that $\left\langle S, \pi_{2}, y\right\rangle=0$ for almost all $y \in V$.

To prove the claim, we fix $C^{2}$-cell decompositions of $V \oplus V$ and $V$ which are compatible with $\pi_{2}$ and spt $S$. If the conclusion does not hold, there exist a cell $D$ of $V$ of dimension $n$, finitely many pairwise and pointwise different definable $C^{2}$ functions $f_{1}, \ldots, f_{k}: D \rightarrow V$ and non-vanishing natural numbers $a_{i}$ such that

$$
\left\langle S, \pi_{2}, y\right\rangle=\sum_{i=1}^{k} a_{i} \delta_{\left(f_{i}(y), y\right)} \quad \forall y \in D .
$$

For almost all $y \in D$ we have $h_{S}(y)=\sum_{i=1}^{k} a_{i} \delta_{\left\langle f_{i}(y), y\right\rangle}=0$. This implies that $k>1$ and that there exists some index $i \neq 1$ with $\left\langle f_{i}(y), y\right\rangle=\left\langle f_{1}(y), y\right\rangle$. We thus find an open subset $D^{\prime}$ of $D$ and an index $i \neq 1$, such that $\left\langle f_{i}(y), y\right\rangle=\left\langle f_{1}(y), y\right\rangle$ for all $y \in D^{\prime}$.

Let $y \in D^{\prime}$. The Legendrian condition implies that $\left\langle d f_{1}(v), y\right\rangle=\left\langle d f_{i}(v), y\right\rangle=$ 0 for all $v \in T_{y} D^{\prime}$. Setting $v:=f_{1}(y)-f_{i}(y) \neq 0$ we obtain that

$$
\underbrace{\left.\frac{d}{d t}\right|_{t=0}\left\langle f_{1}(y+t v)-f_{i}(y+t v), y+t v\right\rangle}_{=0 \text { since } y+t v \in D^{\prime} \text { for small } t}=\underbrace{\left\langle f_{1}(y)-f_{i}(y), v\right\rangle}_{=\langle v, v\rangle \neq 0} .
$$

This is a contradiction and finishes the proof of the claim.

Let $m$ be the dimension of the projection $\pi_{2}(\operatorname{spt} S)$. Then $m<n$, since $\left\langle S, \pi_{2}, y\right\rangle=0$ for almost all $y \in V$. We may choose coordinates in such a way that $\operatorname{dim} \psi \circ \pi_{2}(\operatorname{spt} S)=m$, where $\psi: V \rightarrow \mathbb{R}^{m}$ denotes projection on the first $m$ coordinates.

Suppose $S \neq 0$. Fix compatible $C^{2}$-cell decompositions of spt $S, \pi_{2}(\operatorname{spt} S)$ and $\psi \circ \pi_{2}(\operatorname{spt} S)$. Let $D^{\prime}$ be an $m$-dimensional cell of $\psi \circ \pi_{2}(\operatorname{spt} S)$. A cell 
$D \subset \pi_{2}(\operatorname{spt} S)$ with $\psi(D)=D^{\prime}$ is a graph, since bands would have dimensions strictly larger than $m$. It follows that

$$
A:=\psi^{-1}\left(y^{\prime}\right) \cap \pi_{2}(\operatorname{spt} S) \subset V
$$

is finite for almost all $y^{\prime} \in D^{\prime}$.

The slice $\left\langle S, \psi \circ \pi_{2}, y^{\prime}\right\rangle$ is a non-vanishing definable cycle with support in $V \times A$. For some $y \in A$, its restriction $R$ to $V \times\{y\}$ is again a non-vanishing definable cycle. Let $D$ be the cell containing $y$.

The cell decomposition of spt $S$ induces a natural cell decomposition of spt $R$, with cells being the intersections $\tilde{D}_{y}:=\tilde{D} \cap(V \times\{y\})$, where $\tilde{D}$ runs over all cells of spt $S$.

Let $\tilde{D} \subset \operatorname{spt} S$ be an $n$-dimensional cell with $\tilde{D}_{y} \neq \emptyset$. Then $D=\pi_{2}(\tilde{D})$.

Let $v \in T_{(x, y)} \tilde{D}_{y}$ be a tangent vector. Since $\tilde{D}$ is Lagrangian and $T_{(x, y)} \tilde{D}_{y} \subset$ $T_{(x, y)} \tilde{D}$, it follows that

$$
\left\langle d \pi_{1}(v), d \pi_{2}(w)\right\rangle=\left\langle d \pi_{1}(v), d \pi_{2}(w)\right\rangle-\langle\underbrace{d \pi_{2}(v)}_{=0}, d \pi_{1}(w)\rangle=\omega(v, w)=0
$$

for all $w \in T_{(x, y)} \tilde{D}$. In other words, $d \pi_{1}(v)$ is orthogonal to $T_{y} D$.

Let $\phi: V \rightarrow T_{y} D$ denote orthogonal projection. Then the rank of $\phi \circ \pi_{1}$, restricted to $\tilde{D}_{y}$, is 0 , which implies that there exists a finite set $B \subset T_{y} D$ with

$$
\tilde{D}_{y} \subset \phi^{-1} B \times\{y\} .
$$

Since this is true for all $\tilde{D}$ as above (where $B$ may differ), $R$ is a non-vanishing definable $n-m$-cycle with support contained in a finite disjoint union of $n-m$-dimensional affine subspaces. This contradicts the constancy Theorem 4.4 .

\section{Properties of the normal cycle}

7.1. Projections. Let $W \subset V$ be an oriented linear subspace of dimension $l, W^{\perp}$ its orthogonal complement, oriented in such a way that $W^{\perp} \oplus W$ has the same orientation as $V$, and let $\pi_{W}: V \rightarrow W$ and $\pi_{W^{\perp}}: V \rightarrow W^{\perp}$ be the orthogonal projections.

Proposition 7.1: Let $\phi$ be a compactly supported constructible function on $V$. Then

$$
S_{\left(\pi_{W}\right)_{*} \phi}=\underbrace{\left\langle\left(\pi_{W}, \mathrm{id}\right)_{*} S_{\phi}, \pi_{W^{\perp}} \circ \pi_{2}, 0\right\rangle}_{=: \pi_{W}\left(S_{\phi}\right)} .
$$


The slice on the right hand exists, is supported in $W \oplus W$ and can be considered as a current on $W \oplus W$.

\section{Proof:}

Claim 1: The slice exists.

Let $S:=S_{\phi}$ and

$$
A:=\{(x, y) \in \operatorname{spt} S: y \in W\} .
$$

Let $w_{1}^{\prime}, \ldots, w_{n-l}^{\prime}$ denote an orthogonal base of $W^{\perp}$.

Fix a $C^{2}$-cell decomposition of $A$, a cell $D$ and $(x, y) \in D$. Suppose that $\operatorname{dim} D=d$ and that the vectors $\left(v_{i}, w_{i}\right) \in T_{(x, y)} D, i=1, \ldots, d$ form a base of $T_{(x, y)} D$. Since $S_{\phi}$ is Lagrangian, $\omega\left(\left(v_{i}, w_{i}\right),\left(v_{j}, w_{j}\right)\right)=0$. From $w_{i} \in W$ we infer that $\left\langle v_{j}, w_{i}\right\rangle=\left\langle\pi_{W}\left(v_{j}\right), w_{i}\right\rangle$.

Let $L$ be the subspace generated by the vectors $\left(\pi_{W}\left(v_{i}\right), w_{i}\right), i=1, \ldots, d$. The subspaces $L$ and $\left(\{0\} \times W^{\perp}\right)$ are transversal and their sum is an isotropic subspace of $V \oplus V$, hence of dimension $\leq n$. It follows that

$$
\operatorname{dim} L+\underbrace{\operatorname{dim}\left(\{0\} \times W^{\perp}\right)}_{=n-l} \leq n .
$$

We deduce that $\left.\operatorname{rank}\left(\pi_{W}\right.$, id $)\right|_{D} \leq l$ and thus $\operatorname{dim}\left(\pi_{W}\right.$, id $)(D) \leq l$. Since $\left(\pi_{W}, \mathrm{id}\right)(A)$ is a union of such sets, it has dimension $\leq l$. It follows that

$$
\operatorname{dim}\left(\left(\operatorname{spt}\left(\pi_{W}, \mathrm{id}\right)_{*} S\right) \cap\left(\pi_{W \perp} \circ \pi_{2}\right)^{-1}(0)\right) \leq l,
$$

which implies (by Proposition 4.5) that the slice on the right hand side of (2) exists.

Claim 2: $\pi_{W}(S)$ is a definable conical Lagrangian cycle in $W \oplus W$

From Proposition 4.5 (2) we see that the right hand side of (2) is a definable cycle.

With the notations of Section 4.3 and using 4.5, (3) we see that

$$
\begin{aligned}
\left(m_{\lambda}\right)_{*}\left\langle\left(\pi_{W}, \mathrm{id}\right)_{*} S_{\phi}, \pi_{W^{\perp}} \circ \pi_{2}, 0\right\rangle & =\left(m_{\lambda}\right)_{*}\left\langle\left(\pi_{W}, \mathrm{id}\right)_{*} S_{\phi}, \pi_{W^{\perp}} \circ \pi_{2} \circ m_{\lambda}, 0\right\rangle \\
& =\langle\underbrace{\left(m_{\lambda}\right)_{*} \circ\left(\pi_{W}, \mathrm{id}\right)_{*}}_{=\left(\pi_{W}, \mathrm{id}\right)_{*} \circ\left(m_{\lambda}\right)_{*}} S, \pi_{W^{\perp}} \circ \pi_{2}, 0\rangle \\
& =\left\langle\left(\pi_{W}, \mathrm{id}\right)_{*} S, \pi_{W^{\perp}} \circ \pi_{2}, 0\right\rangle .
\end{aligned}
$$

Hence $\pi_{W}(S)$ is conical.

Since the support of $\pi_{W}(S)$ is contained in $\left(\pi_{W}\right.$, id $)(A)$, the proof of Claim 1 also shows that this current is Lagrangian. 
Claim 3: The support function of $\pi_{W}(S)$ equals $\left.h_{S}\right|_{W}$

We want to apply Proposition 4.5 (4) to the current $\left(\pi_{W}, \text { id }\right)_{*} S$ and the orthogonal projections onto the spaces $V \oplus W$ and $V \oplus W^{\perp}$. We have to check the condition on the dimension. Since $\partial\left(\pi_{W}, \mathrm{id}\right)_{*} S=\partial S=0$, there are only three conditions. The first one is already proved, see Inequality (3).

Since $\operatorname{spt}\left(\pi_{W}, \text { id }\right)_{*} S$ is a definable set of dimension $\leq n$, we get for almost all $y \in W$

$$
\operatorname{dim}\left(\operatorname{spt}\left(\pi_{W}, \mathrm{id}\right)_{*} S \cap\left(\pi_{W} \circ \pi_{2}\right)^{-1}(y)\right) \leq n-l .
$$

Inequality (3) also implies that for almost all $y \in W$

$$
\operatorname{dim}\left(\operatorname{spt}\left(\pi_{W}, \mathrm{id}\right)_{*} S \cap\left(\pi_{W^{\perp}} \circ \pi_{2}\right)^{-1}(0) \cap\left(\pi_{W} \circ \pi_{2}\right)^{-1}(y)\right) \leq 0 .
$$

We can therefore apply Proposition 4.5 (4) to conclude that

$$
\begin{aligned}
\left\langle\left\langle\left(\pi_{W}, \mathrm{id}\right)_{*} S, \pi_{W} \perp\right.\right. & \left.\left.\circ \pi_{2}, 0\right\rangle, \pi_{W} \circ \pi_{2}, y\right\rangle= \\
& \langle\left(\pi_{W}, \mathrm{id}\right)_{*} S, \underbrace{\left(\pi_{W^{\perp}} \circ \pi_{2}, \pi_{W} \circ \pi_{2}\right)}_{=\pi_{2}},(0, y)\rangle=\left(\pi_{W}, \mathrm{id}\right)_{*}\left\langle S, \pi_{2}, y\right\rangle
\end{aligned}
$$

for almost all $y \in W$.

From $u \circ\left(\pi_{W}\right.$, id $)=u$ on $V \oplus W$ we obtain that the support function of the cycle $\left\langle\left(\pi_{W}, \text { id }\right)_{*} S, \pi_{W^{\perp}} \circ \pi_{2}, 0\right\rangle$ equals the support function of $S$ for almost all $y \in W$. Since both functions are Lipschitz continuous (Theorem 5.1), they coincide for all $y \in W$.

\subsection{Products.}

Proposition 7.2: Given oriented Euclidean vector spaces $V$ and $W$ and compactly supported constructible functions $\phi$ on $V$ and $\psi$ on $W$,

$$
S_{\phi \otimes \psi}=S_{\phi} \times S_{\psi} .
$$

Proof: Straightforward using Proposition 2.12 (6).

\subsection{LiNEAR TRANSFORMATIONS AND CONVOLUTION.}

Proposition 7.3: Let $A \in G L(V)$ and $\phi$ a compactly supported constructible function. Then

$$
S_{A_{*} \phi}=\operatorname{sgn}(\operatorname{det} A) \cdot\left(A,\left(A^{*}\right)^{-1}\right)_{*} S_{\phi} .
$$

Proof: Straightforward using Proposition 2.12 (4). 
Proposition 7.4:

(1) Let $\phi, \psi$ be compactly supported constructible functions on $V$. Let $\Delta \subset$ $V \oplus V$ be the diagonal and $\tau: \Delta \rightarrow V,(x, x) \mapsto x$. Then the normal cycle of $\phi * \psi$ is given by

$$
S_{\phi * \psi}=(2 \tau, \tau)_{*} \pi_{\Delta}\left(S_{\phi} \times S_{\psi}\right) .
$$

(2) Let $\psi=1_{B(0, \epsilon)}$ and $\exp ^{\epsilon}: S V \rightarrow S V,(x, v) \mapsto(x+\epsilon v, v)$. Then

$$
T_{\phi * \psi}=\exp _{*}^{\epsilon} T_{\phi} .
$$

Proof:

(1) We do not prove that the current on the right hand side of (4) is a definable, conic Lagrangian cycle, this is a straightforward computation. With $u_{\Delta}: \Delta \times \Delta \rightarrow \mathbb{R},((x, x),(y, y)) \mapsto 2\langle x, y\rangle$ denoting the restriction of the scalar product of $V \oplus V$ to $\Delta$, we have $u \circ(2 \tau, \tau)=u_{\Delta}$.

By Propositions 4.5, 7.1 and 7.2, we have for almost all $y \in V$

$$
\begin{aligned}
h_{(2 \tau, \tau)_{*} \pi_{\Delta}\left(S_{\phi} \times S_{\psi}\right)}(y) & =u_{*}\left\langle(2 \tau, \tau)_{*} \pi_{\Delta}\left(S_{\phi} \times S_{\psi}\right), \pi_{2}, y\right\rangle \\
& =u_{*} \circ(2 \tau, \tau)_{*}\langle\pi_{\Delta}\left(S_{\phi} \times S_{\psi}\right), \underbrace{\pi_{2} \circ(2 \tau, \tau)}_{=\tau \circ \pi_{2}}, y\rangle \\
& =\left(u_{\Delta}\right)_{*}\langle\pi_{\Delta}\left(S_{\phi} \times S_{\psi}\right), \pi_{2}, \underbrace{\left.\tau^{-1}(y)\right\rangle}_{=(y, y)} \\
& =h_{\pi_{\Delta}\left(S_{\phi} \times S_{\psi}\right)}(y, y) \\
& =h_{\phi}(y) \cdot h_{\psi}(y) \\
& =h_{\phi * \psi}(y) .
\end{aligned}
$$

(2) It is easily checked that $\exp _{*}^{\epsilon} T_{\phi}$ is again a definable Legendrian cycle. Its support is contained in the $\epsilon$-neighborhood of the support of $T_{\phi}$, and thus compact. Now for almost all $v \in S(V)$

$$
\begin{aligned}
h_{\exp _{*}^{\epsilon} T_{\phi}}(v) & =u_{*}\left\langle\exp _{*}^{\epsilon} T_{\phi}, \pi_{2}, v\right\rangle \\
& =u_{*} \exp _{*}^{\epsilon}\langle T_{\phi}, \underbrace{\pi_{2} \circ \exp ^{\epsilon}}_{=\pi_{2}}, v\rangle \\
& =\delta_{\epsilon} \cdot u_{*}\left\langle T_{\phi}, \pi_{2}, v\right\rangle \\
& =h_{\psi}(v) \cdot h_{\phi}(v) \\
& =h_{\phi * \psi}(v),
\end{aligned}
$$

which shows that $\exp _{*}^{\epsilon} T_{\phi}$ is the normal cycle of $\phi * \psi$. 
Proposition 7.5: Let $v \in V$ and denote by $\operatorname{tr}_{v}: V \rightarrow V, x \mapsto x+v$ the translation. For a constructible function $\phi$ with compact support,

$$
S_{\phi \circ \mathrm{tr}_{v}}=\left(\operatorname{tr}_{-v}, \mathrm{id}\right)_{*} S_{\phi}
$$

Proof: Straightforward.

\section{Support of the normal cycle}

TheOREM 8.1: Let $\phi: V \rightarrow \mathbb{Z}$ be a constructible function with compact support and $T:=T_{\phi}, S:=S_{\phi}$. Then

(1)

$$
\begin{aligned}
& \pi_{1}(\operatorname{spt} T) \subset\{x \in V: \phi \text { not constant near } x\} \\
& \pi_{1}(\operatorname{spt} S) \subset \operatorname{spt} \phi ;
\end{aligned}
$$

(2) if $\phi$ is constant near $x$, then

$$
\phi(x)=\left[\pi_{1}(T)\right] \in H_{n-1}(V, V \backslash\{x\})=\mathbb{Z} ;
$$

(3) there exists a $C^{2}$-cell decomposition of $\operatorname{spt} \phi$ such that

$$
\operatorname{spt} S_{\phi} \subset \bigcup_{D \text { cell }} \text { Nor } D .
$$

Here Nor $D=\left\{(x, y) \in V \oplus V: x \in D, y \perp T_{x} D\right\}$ denotes the normal space of a cell $D$.

Proof:

(1) Suppose first that $\operatorname{spt} \phi \subset B(0, R)$. By the remark just before the uniqueness proof of Theorem 3.1, $h=h_{\phi}$ is given above each cell by functions whose gradients are bounded by $6 R$. The construction in the proof of Theorem 6.2 can therefore be carried out with $L:=6 R$ and shows that $\pi_{1}\left(\operatorname{spt} T_{\phi}\right) \subset B(0,6 R)$ and $\pi_{1}\left(S_{\phi}\right) \subset B(0,6 R)$

Proposition 7.5 implies that, whenever spt $\phi \subset B(x, R)$ with $x \in V$, $R>0$, then $\pi_{1}(\operatorname{spt} T) \subset B(x, 6 R)$ and $\pi_{1}\left(\operatorname{spt} S_{\phi}\right) \subset B(x, 6 R)$.

Now let $\phi$ be constant, say $a$, near $x \in V$. Then there exists $\epsilon>0$ such that $\phi(y)=a$ for $y \in B(x, \epsilon)$. Set $\phi_{0}:=a 1_{B(x, \epsilon)}$ and let $T_{0}:=T_{\phi_{0}}$, $S_{0}:=S_{\phi_{0}}$.

Since $x \notin \operatorname{spt}\left(\phi-\phi_{0}\right)$, we can use compactness to write $\phi-\phi_{0}$ as a finite $\operatorname{sum} \phi-\phi_{0}=\sum_{i=1}^{k} \phi_{i}$ such that $\operatorname{spt} \phi_{i} \subset \operatorname{spt}\left(\phi-\phi_{0}\right)$ and such that $\operatorname{spt} \phi_{i}$ is contained in some ball $B\left(x_{i}, r_{i}\right)$ with the property that $x \notin B\left(x_{i}, 6 r_{i}\right)$. 
Let $T_{i}:=T_{\phi_{i}}, S_{i}=S_{\phi_{i}}$. Then $\pi_{1}\left(\operatorname{spt} T_{i}\right) \subset B\left(x_{i}, 6 r_{i}\right)$ and $\pi_{1}\left(\operatorname{spt} S_{i}\right) \subset$ $B\left(x_{i}, 6 r_{i}\right)$, i.e. $x \notin \pi_{1}\left(\operatorname{spt} T_{i}\right)$ and $x \notin \pi_{1}\left(\operatorname{spt} S_{i}\right)$.

Since $T-T_{0}=\sum_{i=1}^{k} T_{i}, x \notin \pi_{1}\left(\operatorname{spt}\left(T-T_{0}\right)\right)$. An easy computation shows that, in the case $a \neq 0, \pi_{1}\left(\operatorname{spt} T_{0}\right)=S(x, \epsilon)$. Therefore we obtain $x \notin \pi_{1}(\operatorname{spt} T)$.

If $x \notin \operatorname{spt} \phi$, then $S_{0}=0$. Thus $S=\sum_{i=1}^{k} S_{i}$ and we deduce that $x \notin \pi_{1}(\operatorname{spt} S)$.

(2) Note that $\operatorname{spt}\left(\pi_{1}\left(T_{i}\right)\right) \subset \pi_{1}\left(\operatorname{spt} T_{i}\right)$ is supported in a ball not containing $x$, hence $\left[\pi_{1}\left(T_{i}\right)\right]=0$. Therefore

$$
\left[\pi_{1}(T)\right]=\left[a \pi_{1}\left(T_{0}\right)\right]=a\left[S^{n-1}(x, \epsilon)\right]=\phi(x) .
$$

(3) By (1), we find definable $C^{2}$-cell decompositions of spt $S$ and spt $\phi$, compatible with $\pi_{1}$. We can suppose that all cells of spt $S$ are conical. Let $D^{\prime}$ be such a cell, $(x, y) \in D^{\prime}$ and $D:=\pi_{1}\left(D^{\prime}\right)$. Then there are finitely many vectors $v_{1}, \ldots, v_{d} \in T_{(x, y)} D^{\prime}$ such that $d \pi_{1}\left(v_{i}\right), i=1, \ldots, d$ span $T_{x} D$. Now $0=\alpha\left(v_{i}\right)=\left\langle y, d \pi_{1}\left(v_{i}\right)\right\rangle$, which implies that $y \perp T_{x} D$.

THEOREM 8.2: Let $T=T_{\phi}$ be the normal cycle of the compactly supported constructible function $\phi$. Let $T_{\epsilon}:=\exp _{*}^{\epsilon} T$ be the image of $T$ under the geodesic flow of $S V$ after time $\epsilon>0$. Then for every $x \in V$

$$
\phi(x)=\lim _{\epsilon \rightarrow 0^{+}}\left[\left(\pi_{1}\right)_{*} T_{\epsilon}\right] \in H_{n-1}(V, V \backslash\{x\}) .
$$

Proof: By Proposition $7.4(2), T_{\epsilon}$ is the normal cycle of the convolution $\phi_{\epsilon}:=$ $\phi * 1_{B(0, \epsilon)}$. For all $z \in V$

$$
\phi_{\epsilon}(z)=\int_{V} \phi(y) \underbrace{1_{B(0, \epsilon)}(z-y)}_{=1_{B(z, \epsilon)}(y)} d \chi(y)=\chi(\phi \cap B(z, \epsilon)) .
$$

The local conical structure of definable sets ([12], Thm. 4.10, [27]) implies that the right hand side converges to $\phi(z)$ as $\epsilon$ tends to 0 , i.e. $\phi_{\epsilon} \rightarrow \phi$ pointwise. Using Thom's isotopy lemma ([25]) we get that, for all small enough $\epsilon>0, \phi_{\epsilon}$ is constant near $x$. From Theorem 8.1 it follows $x \notin \pi_{1}\left(\operatorname{spt}\left(T_{\epsilon}\right)\right)$ and

$$
\phi_{\epsilon}(x)=\left[\left(\pi_{1}\right)_{*} T_{\epsilon}\right]
$$

Letting $\epsilon$ tend to 0 on both sides finishes the proof.

Let $\rho_{x}: V \backslash\{x\} \rightarrow S(x, 1)$ be the radial projection and $\rho_{x}^{*} d v$ be the pull-back of the volume form on $S(x, 1)$. Then for any cycle $A$ on $V$ with support in 
$V \backslash\{x\}$ we have

$$
[A]=\frac{1}{s_{n-1}} A\left(\rho_{x}^{*} d v\right) .
$$

Here $s_{n-1}$ is the volume of the $n$-1-dimensional sphere.

It follows from the previous theorem that

$$
\phi(x)=\frac{1}{s_{n-1}} \lim _{\epsilon \rightarrow 0^{+}} T\left(\left(\rho_{x} \circ \pi_{1} \circ \exp ^{\epsilon}\right)^{*} d v\right) .
$$

As our argument above shows, the support of $T$ is, for small $\epsilon>0$, disjoint from the singular set of the differential form $\left(\rho_{x} \circ \pi_{1} \circ \exp ^{\epsilon}\right)^{*} d v$ (which is given by the set $\{(z, v) \in S V: z+\epsilon v=x\})$.

Example: Let $X \subset V$ be a compact, definable submanifold. Theorem 8.2 and some elementary topological arguments imply that the normal cycle of $T$ is given by integration over the unit normal bundle of $X$ (which carries a canonical orientation). Another way to see this is to use Morse theory, see [24]. Similarly, using stratified Morse theory ([19]), one can show that the normal cycle of a definable compact subset of $V$ can be described explicitly in terms of Morse indices associated to height functions, see [11].

\section{References}

[1] S. Alesker, Theory of valuations on manifolds, I. Linear Spaces, Israel Journal of Mathematics 156 (2006), 311-340.

[2] S. Alesker, Theory of valuations on manifolds, II, Advances in Mathematics 207 (2006), 420-454.

[3] S. Alesker, Theory of valuations on manifolds, IV, New Properties of the multiplicative structure, 2005, Preprint.

[4] S. Alesker and J. H. G. Fu, Theory of valuations on manifolds, III, Multiplicative structure in the general case, 2005, Preprint.

[5] A. Bernig, Variation of curvatures of subanalytic spaces and Schläfli-type formulas, Annals of Global Analysis and Geometry 24 (2003), 67-93.

[6] A. Bernig, Legendrian Currents, Support Functions and Tensor-Valued Measures on Singular Spaces, Habilitationsschrift, Université de Fribourg, 2005.

[7] A. Bernig, Curvature tensors of singular spaces, Differential Geometry and its Applications 24 (2006), 191-208.

[8] A. Bernig, Support functions, projections and Minkowski addition of Legendrian cycles, Indiana University Mathematical Journal 55 (2006), 443-464.

[9] A. Bernig and L. Bröcker, Valuations on manifolds and Rumin cohomology, Journal of Differential Geometry 75 (2007), 433-457. 
[10] L. Bröcker, Euler integration and Euler multiplication, Advances in Geometry 5 (2005), 145-169.

[11] L. Bröcker and M. Kuppe, Integral geometry of tame sets, Geometriae Dedicata 82 (2000), 285-323.

[12] M. Coste, An Introduction to o-Minimal Geometry, Universitá di Pisa, Dipartimento di Matematica, 2000.

[13] H. Federer, Geometric Measure Theory, Springer-Verlag, New York, 1969.

[14] J. H. G. Fu, Curvature measures and Chern classes of singular varieties, Journal of Differential Geometry 39 (1994), 251-280.

[15] J. H. G. Fu, Curvature measures of subanalytic sets, American Journal of Mathematics 116 (1994), 819-880.

[16] J. H. G. Fu, Some remarks on Legendrian rectifiable currents, Manuscripta Mathematica 97 (1998), 175-187.

[17] J. H. G. Fu and C. McCrory, Stiefel-Whitney classes and the conormal cycle of a singular variety, Transactions of the American Mathematical Society 349 (1997), 809-835.

[18] C. G. Gibson, K. Wirthmüller, A. A. du Plessis and E. J. N. Looijenga, Topological Stability of Smooth Mappings, LNM 552, Springer, Berlin, 1970.

[19] M. Goresky and R. MacPherson, Stratified Morse Theory, Springer, Berlin, 1988.

[20] R. Hardt, Slicing and intersection theory for chains associated with real analytic varieties, Acta Mathematica 129 (1971), 57-136.

[21] R. Hardt, Topological properties of subanalytic sets, Transactions of the American Mathematical Society 211 (1975), 57-70.

[22] M. Kashiwara and P. Shapira, Sheaves on Manifolds, Springer, Berlin, 1994.

[23] M. Llorente and S. Winter, A notion of Euler characteristic for fractals, Mathematische Nachrichten 208 (2007), 152-170.

[24] J. Milnor, Morse Theory, Annals of Mathematics Studies 51, Princeton University Press, Princeton, N.J., 1963.

[25] M. Pflaum, Analytic and Geometric Study of Stratified Spaces, Springer-Verlag, Berlin-Heidelberg, 2001.

[26] R. Schneider, Convex Bodies: the Brunn-Minkowski Theory, Cambridge University Press, Cambridge, 1993.

[27] L. van den Dries, Tame Topology and o-Minimal Structures, Cambridge University Press, Cambridge, 1998.

[28] L. van den Dries and C. Miller, Geometric Categories and o-minimal structures, Duke Mathematical Journal 84 (1996), 497-540. 